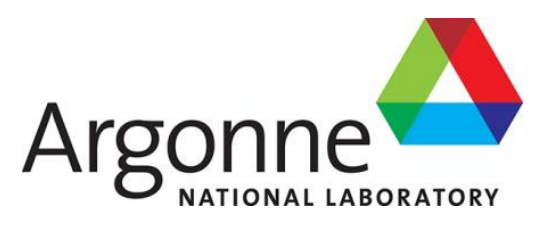

ANL/NE-18/7

\title{
Ultrasonic Link Model Development
}

Transmission of information by acoustic communication along metal pathways in nuclear facilities

FY 2018 Progress Report

Nuclear Engineering Division 


\section{About Argonne National Laboratory}

Argonne is a U.S. Department of Energy laboratory managed by UChicago Argonne, LLC under contract DE-AC02-06CH11357. The Laboratory's main facility is outside Chicago, at 9700 South Cass Avenue, Argonne, Illinois 60439. For information about Argonne and its pioneering science and technology programs, see www.anl.gov.

\section{Document availability \\ Online Access: U.S. Department of Energy (DOE) reports produced after 1991 and a growing number of pre-1991 documents are available free at OSTI.GOV (http://www.osti.gov/), a service of the U.S. Dept. of Energy's Office of Scientific and Technical Information}

\section{Reports not in digital format may be purchased by the public from the} National Technical Information Service (NTIS):

U.S. Department of Commerce

National Technical Information Service

5301 Shawnee Rd

Alexandria, VA 22312

www.ntis.gov

Phone: (800) 553-NTIS (6847) or (703) 605-6000

Fax: (703) 605-6900

Email: orders@ntis.gov

Reports not in digital format are available to DOE and DOE contractors from the Office of Scientific and Technical Information (OSTI):

U.S. Department of Energy

Office of Scientific and Technical Information

P.O. Box 62

Oak Ridge, TN 37831-0062

www.osti.gov

Phone: (865) 576-8401

Fax: (865) 576-5728

Email: reports@osti.gov

\section{Disclaimer}

This report was prepared as an account of work sponsored by an agency of the United States Government. Neither the United States Government nor any agency thereof, nor UChicago Argonne, LLC, nor any of their employees or officers, makes any warranty, express or implied, or assumes any legal liability or responsibility for the accuracy, completeness, or usefulness of any information, apparatus, product, or process disclosed, or represents that its use would not infringe privately owned rights. Reference herein to any specific commercial product, process, or service by trade name, trademark, manufacturer, or otherwise, does not necessarily constitute or imply its endorsement, recommendation, or favoring by the United States Government or any agency thereof. The views and opinions of document authors expressed herein do not necessarily state or reflect those of the United States Government or any agency thereof, Argonne National Laboratory, or UChicago Argonne, LLC. 


\section{Ultrasonic Link Model Development}

Transmission of information by acoustic communication along metal pathways in nuclear facilities

FY 2018 Progress Report

prepared by

A. Heifetz ${ }^{1}$, R. Ponciroli ${ }^{1}$, X. Huang ${ }^{1,2}$, B. Wang ${ }^{1,2}$, J. Saniie ${ }^{2}$, S. Bakhtiari ${ }^{1}$, and R.B Vilim ${ }^{1}$

${ }^{1}$ Nuclear Science and Engineering Division, Argonne National Laboratory

2Department of Electrical and Computer Engineering, Illinois Institute of Technology, Chicago, IL

March 30, 2018 


\section{ABSTRACT}

The objective of this project is to develop and demonstrate methods for transmission of information in nuclear facilities across physical barriers as acoustic/elastic guided waves along existing in-place metal piping infrastructure. Acoustic communication (AC) system hardware and software layers for efficient and resilient transfer of data are being developed, with the goal of performing experimental demonstration of information transmission in a representative environment.

Research efforts in the current reporting period have focused ultrasonic link model development. System design considerations included analysis of communication system power consumption, operational condition requirements, data transfer requirements, and analysis of anticipated losses in the communication channel. Model assessment included software defined ultrasonic communication system development based on Red Pitaya FPGA (field programmable gate arrays) boards. Development of EMAT (electromagnetic acoustic transducers) - based ultrasonic communication hardware consisted of analysis of signal transmission with Lamb wave and shear wave EMAT to PZT (piezoelectric transducer) receiver. 


\section{TABLE OF CONTENTS}

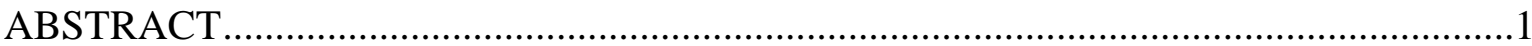

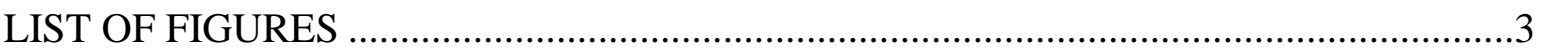

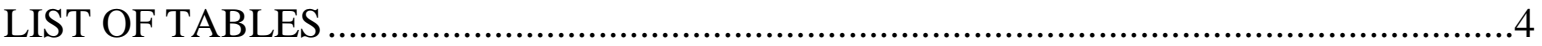

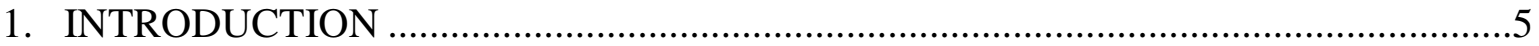

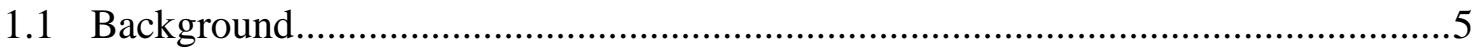

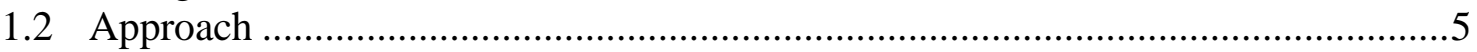

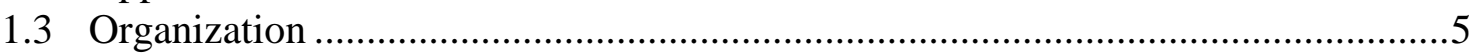

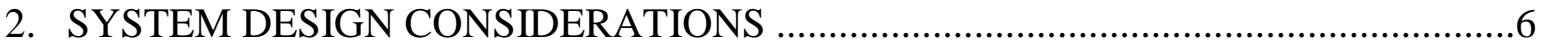

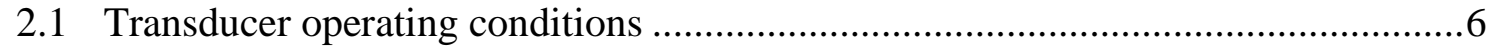

2.2 Reactor process variables and sensors ...........................................................

2.2.1 Cold Leg Temperature: ..............................................................................

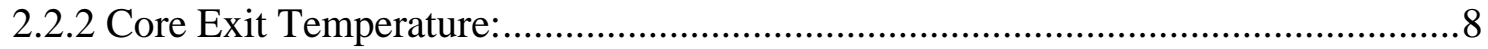

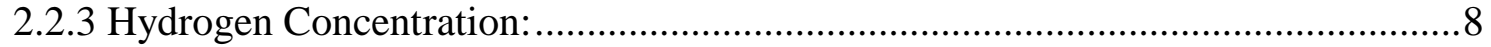

2.3 Data transmission and power consumption ......................................................

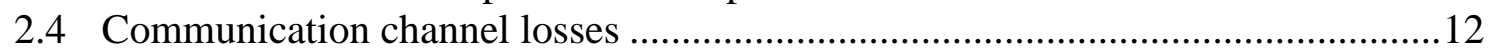

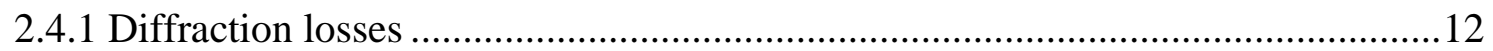

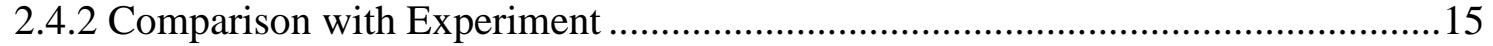

3. SOFTWARE DEFINED ULTRASONIC COMMUNICATION SYSTEM .................17

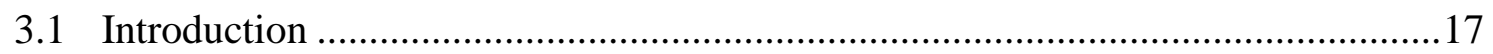

3.2 Hardware Platform .................................................................................. 18

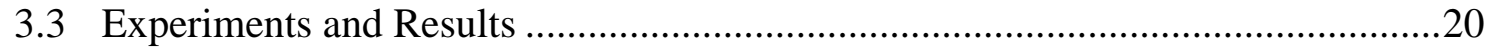

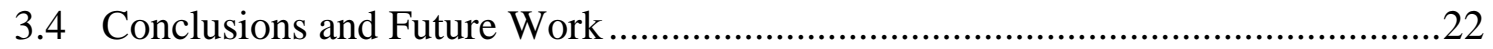

4. EMAT-BASED ULTRASONIC COMMUNICATION SYSTEM ...........................23

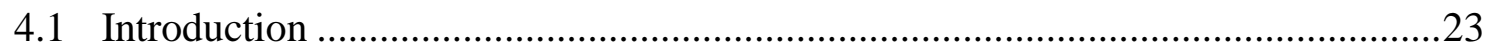

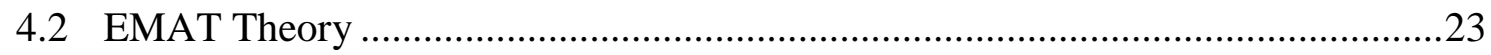

4.3 System Description and Experimental Setup ................................................24

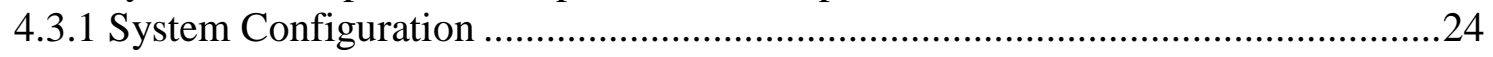

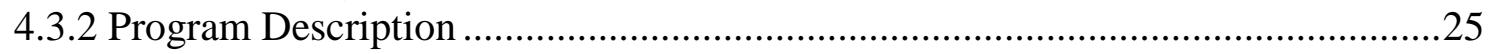

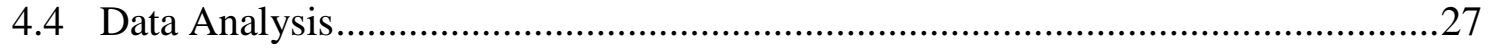

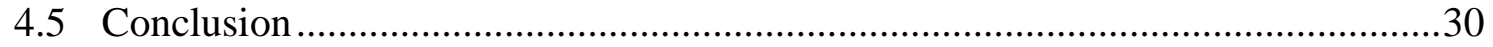

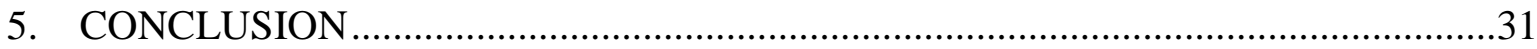

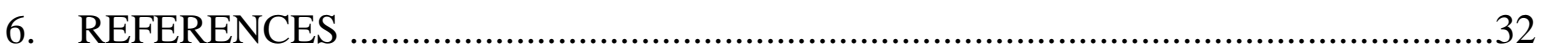




\section{LIST OF FIGURES}

Figure 1. Visualization of data transmission requirements.................................................... 9

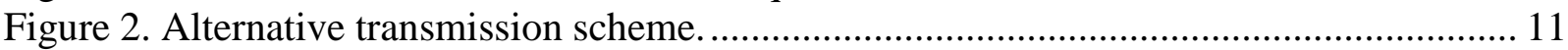

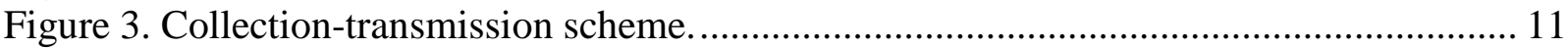

Figure 4. Schematic diagram of acoustic signal transmission................................................. 14

Figure 5. Schematic diagram of acoustic wave refraction and shear wave propagation in

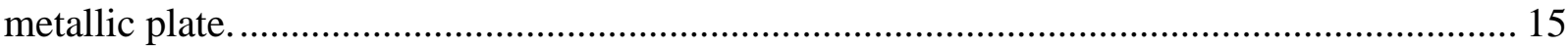

Figure 6. Comparison of calculated and measured acoustic shear wave transmitted signals.... 17

Figure 7. IQ Modulator and De-Modulator block diagrams ................................................. 18

Figure 8. Ultrasonic communication system diagram. ....................................................... 19

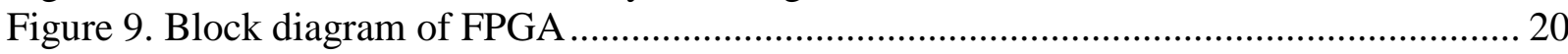

Figure 10. Test Setup A - Transmitting and receiving with transducers at the ends of a column

Figure 11. Test Setup B - Transmitting and Receiving with transducers at oblique angles ..... 21

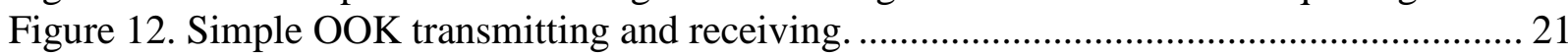

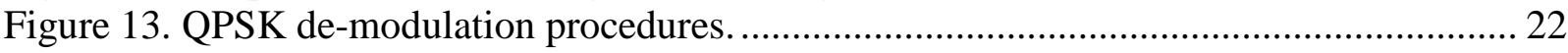

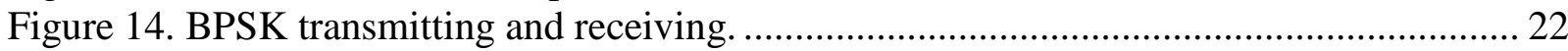

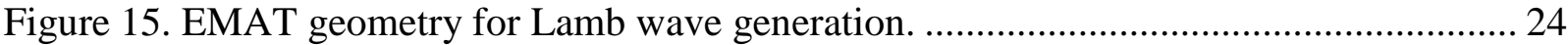

Figure 16. Schematics of laboratory electronics setup......................................................... 25

Figure 17. Communication over stainless steel channels (a) Plate. (b) Pipe. ........................... 25

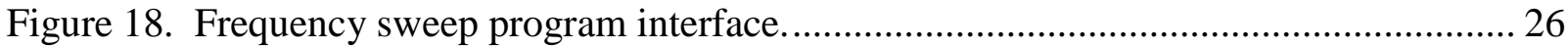

Figure 19. Labview ${ }^{\mathrm{TM}}$ interface of communication system program................................... 27

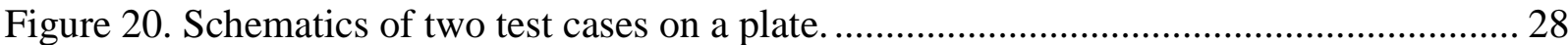

Figure 21. Test results for the plate channel (1) transmitted signal (2) received signal when receiver is $145 \mathrm{~cm}$ away from transmitter (3) received signal when receiver is $120 \mathrm{~cm}$ away from transmitter (4) received signal when receiver is $95 \mathrm{~cm}$ away from transmitter (5) received signal when receiver is $70 \mathrm{~cm}$ away from transmitter (6) received signal when receiver is $35 \mathrm{~cm}$ away from transmitter.

Figure 22. Test results for the pipe channel (1) transmitted signal (2) received signal when receiver is $145 \mathrm{~cm}$ away from transmitter (3) received signal when receiver is $120 \mathrm{~cm}$ away from transmitter (4) received signal when receiver is $95 \mathrm{~cm}$ away from transmitter $\mathrm{m}(5)$ received signal when receiver is $70 \mathrm{~cm}$ away from transmitter (6) received signal when receiver is $35 \mathrm{~cm}$ away from transmitter.

Figure 23. Transmitted and received signals with the plate channel (a) Bit duration of 100 microseconds (b) Bit duration of 50 microseconds (c) Bit duration of 33 microseconds (d) Bit

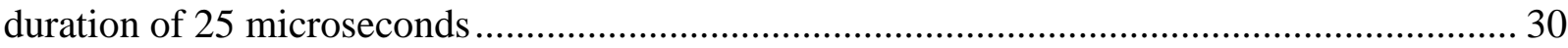

Figure 24. Transmitted and received signals with the pipe channel (a) Bit duration of 100 microseconds (b) Bit duration of 50 microseconds (c) Bit duration 33 microseconds (d) Bit duration of 25 microseconds 


\section{LIST OF TABLES}

Table 1. Typical Environmental Stresses on CI Function Components [3] ............................... 7

Table 2. List of key sensors and corresponding parameters. ……........................................... 8

Table 3. Geometrical parameters of stainless steel pipe.................................................... 15

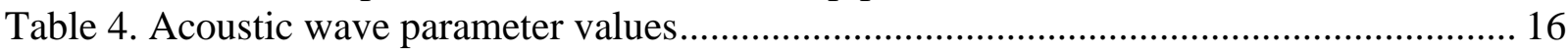

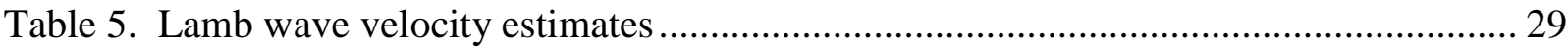




\section{INTRODUCTION}

\subsection{Background}

This project aims to develop and demonstrate methods for transmission of information in nuclear facilities as guided acoustic/elastic waves along existing in-place metallic piping infrastructure. This innovative means of transmitting information overcomes physical hurdles that beset conventional communication methods (both wired and radio frequency (RF) wireless). While the use of wireless RF signals for the transmission and reception of data in nuclear facilities provides, in principle, greater data transfer rate per unit cost, the presence of physical boundaries presents a major challenge to actual implementation. The typical nuclear facility for safety reasons (e.g. confinement of radiation and radionuclides) is heavily partitioned and equipmentpacked resulting in transmission paths that are highly attenuating for electromagnetic waves. Primary barriers include a containment building's thick reinforced high-strength concrete walls, which in some plant designs have liners (steel plates) on the interior side. Additional securityrelated concerns related to use of RF exist because of long distance propagation of RF signal outside of nuclear facility boundaries. Acoustic transmission of information over metallic pipe penetrating containment vessel provides the means for monitoring reactor condition following a severe accident, when all other communication options are disabled. We are currently developing the acoustic communication (AC) hardware and network protocols for efficient and secure transfer of data, and will provide a preliminary experimental demonstration of the AC system prototype in a representative environment.

\subsection{Approach}

The approach makes use of in-place metal process-fluid conduits as the backbone of the physical layer of the AC system. Pipes are omnipresent in a nuclear facility given their role of transferring mass and energy between the outside world and the inner workings of the facility. Piping networks will serve as conduits for signals launched as guided acoustic surface waves. Acoustic transducers compatible with harsh operating environment will be developed, along with efficient digital and analog data communication protocols. The AC system to be developed will be compatible with RF wireless networks due to availability of acoustic to RF transducers. Initial efforts on the project have focused on identification of appropriate transducers and coupling mechanism for transmission and reception of acoustic signals. In particular, the performance bounds on information transmission along a pipe using a pair of commercially available PZTs (piezo electric transducers) and EMATs (electromagnetic acoustic transducers) were considered to obtain figure-of-merit estimates. Although other types of transducers are available, PZT's and EMAT's are some of the most common in use, primarily for NDE applications.

\subsection{Organization}

This report describes research efforts that focused on development of ultrasonic links model. Section 2, System Design Considerations, discusses, transducer operational conditions, data transfer requirements which account for sensor time constants, and communication system power consumption, and communication channel losses. Section 3, Software Defined Ultrasonic Communication System, discusses architecture of ultrasonic system utilizing Red Pitaya FPGA boards. Section 4, EMAT-Based Ultrasonic Communication System, discusses analysis of signal 
transmission with Lamb Wave and shear wave EMATs. Section 5, Conclusions, summarizes the results of this study. Section 6 , References, provides a bibliography for the material in this report.

\section{SYSTEM DESIGN CONSIDERATIONS}

\subsection{Transducer operating conditions}

Acoustic communication system under development in this project is intended to provide accident-resilient communication option for the nuclear facility. That is, the communication system is expected to operate in normal and post-accident scenarios. As previously described [1], during an extended station blackout (SBO), the communication unit cannot rely on unlimited power supply. Therefore, we assumed that the only way the acoustic transmission system can be powered is through a battery, such as battery pack of a Tesla Model S (100kWh). To synchronize our efforts with severe accident response R\&D, we stipulate that communication system is expected to be used in post-accident reactor monitoring for at least 72 hours. Acoustic wave transducers considered in this project include PZT (piezo electric transducers) and EMAT (electromagnetic acoustic transducers). Although PZT consumes less power, EMAT is advantageous for applications in high radiation and temperature environment. In addition, as will be discussed below, EMAT has wideband response (100\% 3dB points of the center frequency), as compared to narrowband PZT (10\% 3dB points of the center frequency). This potentially allows to use frequency modulation (FM) communication protocol with EMAT transducers

As discussed in the previous reports, the acoustic transmission scheme is going to be installed on a pipe which is associated with the auxiliary systems. As reported in [1], the CVCS charging lines inside containment are usually insulated. For example, in the Calvert Cliffs units, the insulation material for the charging line is mineral wool in panels [2]. The panels typically consist of 22 gauge stainless steel sheet, a thickness of 9 pound density mineral wool (corresponding to about 2 inch thickness), and a 24 gauge stainless steel sheet. In addition to being thermal insulator, mineral wool is also a strong attenuator for ultrasonic waves.

All pipes installed in a nuclear unit are sized and certified as compliant with the appropriate ASME codes. Accordingly, once a pressure boundary of pipe is certified, any intervention that might affect its capability to sustain normal operation pressure transients is not allowed. Therefore, any tampering with pipe surface, such as welding and drilling of the pipe is not allowed. On the other hand, there are no explicit statements against locally removing a section of the mineral wool to allow the installation of transducers. Minimal heating losses would be expected from removal of small section of insulation

The charging line of CVCS (chemical and volume control system) was previously identified as a promising location for the application of an acoustic transmission device. This is because only the charging line offers the combination of two favorable characteristics: a relatively low temperature and containment isolation valves both placed outside containment. These conditions refer to the normal operation. To be conservative, the accidental conditions should be accounted for as well. The acoustic transmission system is expected to be particularly useful when other conventional communication means cannot be used. Therefore, theoretically, the worst accidental scenario conditions (BDBA, Beyond Design Basis Accident) should be accounted for. Otherwise, at this preliminary stage, we will consider DBA (Design Basis Accident) conditions only. The transducers are expected to be applied to the CVCS charging line inside the containment. The components in the primary containment are exposed to the most severe 
temperature, humidity, and radiation environments. Typical value of environmental stresses of containment isolation (CI) function components inside the PWR containment [3] are listed in Table 1. We assume that the transducers are subject to the same environmental stresses that are experienced by the $\mathrm{CI}$ function components inside the primary containment.

Table 1. Typical Environmental Stresses on CI Function Components [3]Error! Reference source not found.

\begin{tabular}{ccc}
\hline Parameter & Normal & Accident \\
\hline Temperature & $50-120^{\circ} \mathrm{F}$ & $300{ }^{\circ} \mathrm{F}$ \\
Pressure & atmospheric & $70 \mathrm{psig}, \max$ \\
Relative Humidity & $30-100 \%$ & $100 \%$ \\
Radiation & $50 \mathrm{rads} / \mathrm{hr}$ & $150 \mathrm{Mrads} / \mathrm{hr}$ \\
\hline
\end{tabular}

Therefore, following DBA conditions, we can assume design temperature of $150{ }^{\circ} \mathrm{C}$, as well as high humidity and elevated ionizing radiation levels for the acoustic transducers operational environment.

Given the expected operating conditions, we conclude that despite higher power consumption, EMAT is preferable for implementation of information transmission system at the power plant. Because of relative simplicity, PZT is used for proof-of-principle communication system development. However, eventual design is going to consist of EMAT transmitter and receiver.

\subsection{Reactor process variables and sensors}

Estimates of communication system power requirements consist of surveying critical reactor process variables, information on which needs to be transferred outside the containment building in a post-accident scenario. Data requirements for transmitting information from these sensors constitute the minimum set of requirements for a viable communication system at a nuclear facility. Table 2 list the reactor process variables, and corresponding measurement sensors. These sensors are characterized by specific time constants, i.e. each sensor has a specific dynamic behavior which will contribute determining the frequency of the transmitted train signals [4], [5].

The sensors generate current or voltage signals $(\mathrm{mV}$ or $\mathrm{mA})$ that can be converted into the corresponding units of measurement $\left({ }^{\circ} \mathrm{C}\right.$, bar, ...) before being transmitted. On the other hand, the number of bits corresponding to each one of the monitored variables depends on the desired resolution, which is characteristic of the considered variable. Therefore, the number of dedicated bits does not depend on the features of the sensors under consideration, but on the A/D converter. In common practice, using 24 bits will ensure a very high resolution. Currently, the standard A/D converters use 8-16 bits. For our purposes, as a first guess, we will assume that all the monitored variables are encoded by using 16 bits.

Comments below provide a slightly expanded discussion of the parameters of a few select process variables and corresponding measurement sensors: 


\subsubsection{Cold Leg Temperature:}

PWR plants often employ 20 to 40 RTDs to measure the fluid temperature in the reactor coolant system. RTDs are used in PWR plants to measure the primary coolant temperature. Typically, these RTDs are installed in thermowells that are welded to the primary coolant piping [6]Error! Reference source not found.. The temperatures measured by the RTDs are used by the plant operators for process control and to assess the operational status and safety of the plant.

\subsubsection{Core Exit Temperature:}

In addition to the RTDs, there are also about 50 core-exit thermocouples (CETs) in PWRs to provide an additional way to monitor reactor coolant temperature. Accuracy for CETs is not as important as for RTDs because CETs are used mostly for temperature monitoring. In each loop of a PWR plant and for each core quadrant, redundant RTDs and CETs are used to minimize the probability of failure of any one RTD or CET affecting the safety of the plant.

\subsubsection{Hydrogen Concentration:}

The concentration of hydrogen within the containment is assumed to be measured by using a palladium-based thick film sensor [7]. The characteristic response times are equal to 20-30 seconds, and the recovery times are of the same magnitude. This slow dynamics is due to the long time constants associated with the diffusion of hydrogen into the palladium layer, and the effects of temperature.

Table 2. List of key sensors and corresponding parameters.

\begin{tabular}{|c|c|c|c|c|c|}
\hline Variable & Sensor & $\begin{array}{c}\text { Time } \\
\text { constant (s) }\end{array}$ & $\begin{array}{l}\text { Signal } \\
\text { Range }\end{array}$ & $\begin{array}{c}\text { Variable } \\
\text { Range }\end{array}$ & \# bits \\
\hline Control Rod Position & Linear transducer & - & - & - & 16 \\
\hline Cold Leg Temperature & TC (K-type) & 3 & $0-78 \mathrm{mV}$ & $0-1250{ }^{\circ} \mathrm{C}$ & 16 \\
\hline Core Exit Temperature & $\mathrm{TC}$ & 3 & $0-78 \mathrm{mV}$ & $0-1250{ }^{\circ} \mathrm{C}$ & 16 \\
\hline RCS Pressure & $\begin{array}{l}\text { Strain pressure } \\
\text { transducer }\end{array}$ & 0.25 & $0-20 \mathrm{~mA}$ & $0-500 \mathrm{~Pa}$ & 16 \\
\hline RCS Inventory & $\begin{array}{c}\text { Differential pressure } \\
\text { transducer }\end{array}$ & $<1$ & $0-20 \mathrm{~mA}$ & $0-500 \mathrm{~Pa}$ & 16 \\
\hline $\begin{array}{l}\text { Containment Sump Water } \\
\text { Level }\end{array}$ & $\begin{array}{l}\text { Differential pressure } \\
\text { transducer }\end{array}$ & $<1$ & $0-20 \mathrm{~mA}$ & $0-500 \mathrm{~Pa}$ & 16 \\
\hline Containment Pressure & $\begin{array}{l}\text { Strain pressure } \\
\text { transducer }\end{array}$ & 0.25 & $0-20 \mathrm{~mA}$ & $0-500 \mathrm{~Pa}$ & 16 \\
\hline $\begin{array}{l}\text { Containment Isolation } \\
\text { Valve Position }\end{array}$ & Potentiometer & $<1$ & $0-5 \mathrm{~V}$ & $0-359^{\circ}$ & 16 \\
\hline Hydrogen Concentration & $\begin{array}{l}\text { Electrochemical } \\
\text { Galvanic cell }\end{array}$ & 30 & - & - & 16 \\
\hline SG level & $\begin{array}{c}\text { Differential pressure } \\
\text { transducer }\end{array}$ & $<1$ & $0-20 \mathrm{~mA}$ & $0-500 \mathrm{~Pa}$ & 16 \\
\hline SG pressure & $\begin{array}{l}\text { Strain pressure } \\
\text { transducer }\end{array}$ & 0.25 & $0-20 \mathrm{~mA}$ & $0-500 \mathrm{~Pa}$ & 16 \\
\hline
\end{tabular}


Figure 1 provides conceptual visualization of data transmission rates. In particular, the upper theoretical bound (Channel Capacity (Shannon Limit)) [8] is derived from the information theory, Eq. (1)

$$
C=W \log _{2}\left(1+\frac{P}{N_{0} W}\right)
$$

The limits imposed by the data collection rates due to the sensor features (Sensor dynamicslimited Channel Capabilities) are more penalizing. Finally, the minimum data rate is the one which allows meeting the minimum monitoring level imposed by the unit safety (Safetyoriented Performance). As previously mentioned, during post-accident scenarios, the system evolution is pretty slow, and we are not expecting the system conditions to abruptly vary. As first tentative guess, we might assume that the system conditions might be updated every minute. This value can be considered as the lower acceptable bound for the data transmission rate for safety-grade monitoring purposes.

$$
\begin{aligned}
& \tau_{\text {safety }}=1 \mathrm{~min} \\
& f^{\text {min }}=f_{\text {safety }}=\frac{1}{\tau_{\text {safety }}}
\end{aligned}
$$

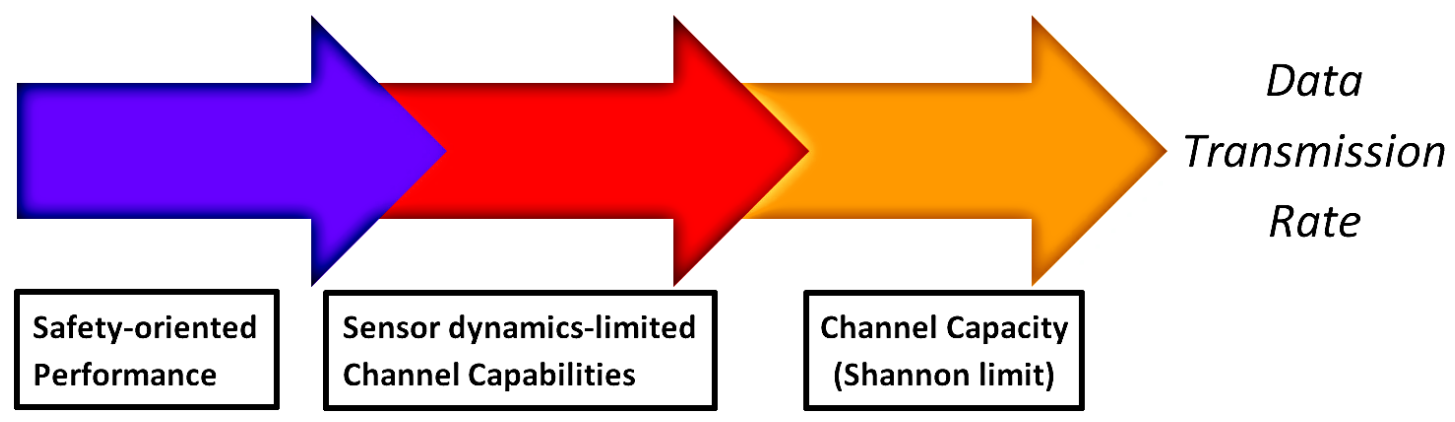

Figure 1. Visualization of data transmission requirements.

\subsection{Data transmission and power consumption}

In development of preliminary data transmission protocols, the following assumptions have been made:

(1) Train of signals, i.e. the sensor outputs are progressively collected and then transmitted at the same time.

(2) The number of bits dedicated to encode the instantaneous value of a certain variable is constant.

(3) As first guess, 16 bits are assumed to be used for all the monitored variables.

(4) PSK communication protocol, i.e. constant power consumption to encode zeros and ones. 
Accordingly, the overall length of the transmitted binary string $(L)$ is equal to the sum of the number of bits you need to encode each one of the $M$ monitored variables together with the 4digits Barker sequence, Eq. (4).

$$
L(t)=L=4+\sum_{j=1}^{M} N_{j} \quad \text { where } j=1,2, \ldots, M
$$

In virtue of assumption (2), the length of the train of signals is always the same. In order to evaluate the energy consumption for every train of signals, the first step is the definition of the number of bits dedicated to encode the variables listed in Table 2. In virtue of assumption (2), we will assume 16 bits for all the monitored variables. Therefore,

$$
L(t)=L=196 \text { bits }
$$

The main consequence of the adoption of the PSK communication protocol (assumption (4)) is that the energy consumption to encode a logic " 1 " or " 0 " $\left(e^{1,0}\right)$ is the same. Therefore, the energy consumption for the transmission of each one of the $M$ variables $(e(j))$ only depends on the number of dedicated bits $(\# b i t(j))$.

$$
e(j)=e^{1,0} \cdot \# \operatorname{bit}(j)
$$

Consequently, every train of signals consumes the same amount of energy. This result tremendously simplifies the problem, since the stochastic nature of power consumption is removed, and more accurate estimates of the sampling frequency bounds according to the power consumption criterion can be derived. As a first guess (assumption (3)), we will have

$$
e_{\text {TRAIN }}(t)=e_{\text {TRAIN }} \cong e^{1,0} \cdot 196
$$

As for the single digit power consumption, the bit pulse is characterized by a minimal length value. In particular, if a pulse is shorter than $100 \mu s$, it is difficult to determine if it is " 1 " or "0"

The proposed approach is based on the collection of all the sensor output signals, which are eventually transmitted at the same time as a train of signals. The sensors listed in Table 2 are characterized by very different time constants. According to the proposed approach, the frequency of transmission $(f)$ is set equal to the reciprocal of the time constant of the slowest dynamics sensor $\left(\tau_{M}\right)$, i.e. 30 seconds. Unfortunately, this frequency might be too low. Therefore, an alternative communication approach, illustrated in Figure 2, could be utilized. Let us set the frequency of transmission equal to the time constant of the second slowest dynamics sensor $\left(\tau_{M-1}\right)$, which is expected to be much lower than 30 seconds. In this way, when the collected measurement outcomes are transmitted, the measurement of the hydrogen concentration will not be complete, and then the previously obtained value will be used. This scheme is not efficient. Therefore, in the proposed approach, the hydrogen conditions will be transmitted only when the measurements have been completed; when they have not, a shorter train signals will be transmitted. 


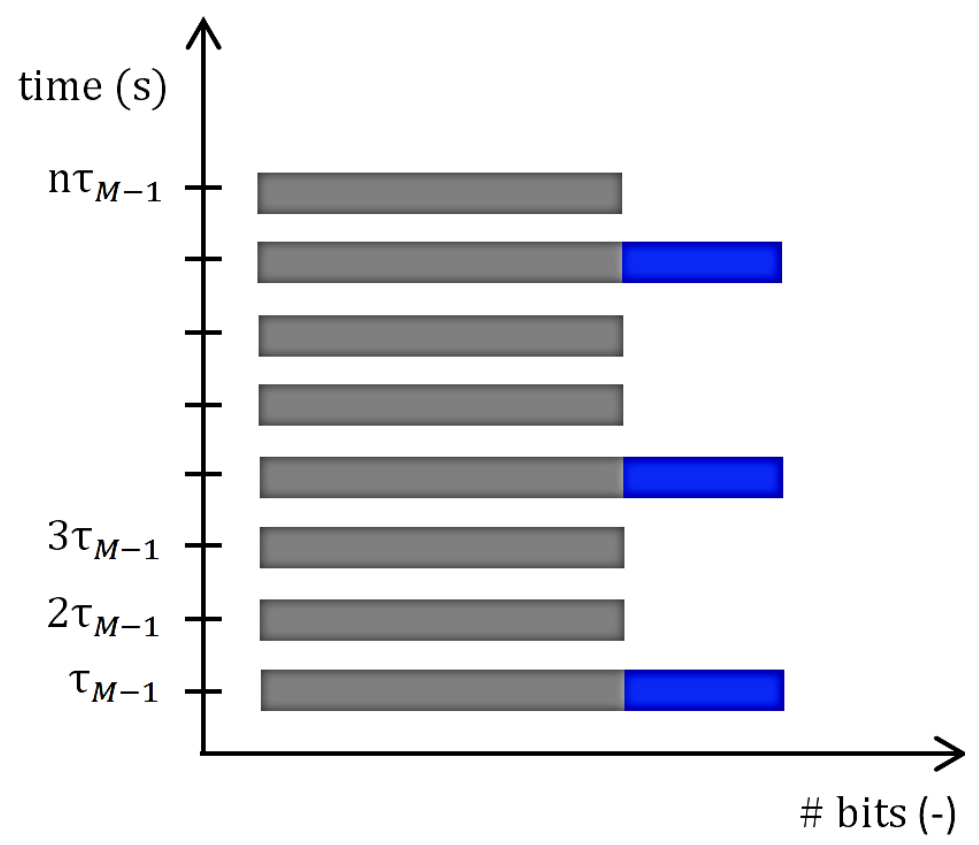

Figure 2. Alternative transmission scheme.

Let us assume that the transmission frequency is equal to $f$. Then, the time can be discretized in $\left(\frac{1}{f}\right)$-long time periods. Each time period is composed by two phases, i.e. the signal collection and the signal transmission, as shown in Figure 3.

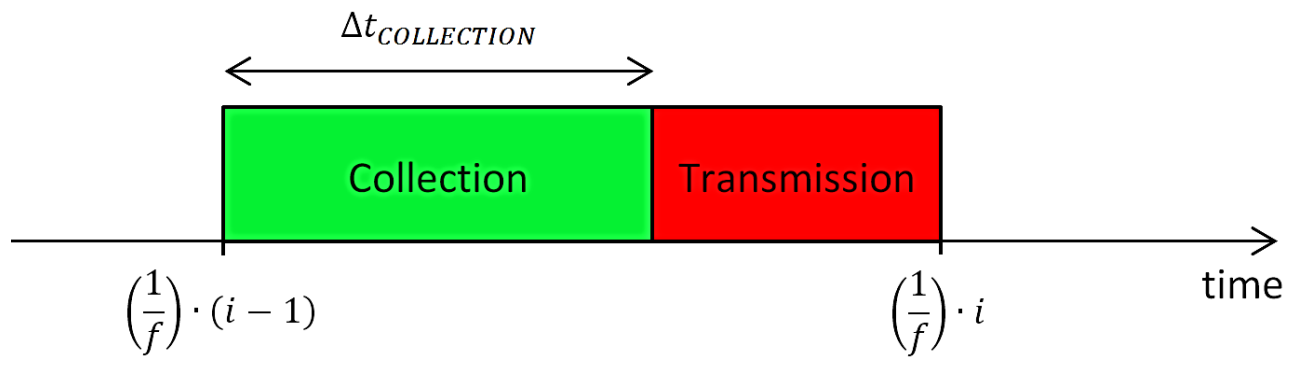

Figure 3. Collection-transmission scheme.

The evaluation of the energy consumed in every time period $(e)$ strongly affects the value of the maximum transmission frequency which is compatible with the battery pack energy availability, Eq. (8).

$$
f^{\max }=\left(\frac{100 \mathrm{kWh}}{72 h}\right) \cdot \frac{1}{e}
$$

The time period energy consumption is given by the sum of the Red Pitaya-related energy consumption during the signal collection phase and the energy consumed to transmit the train of signals, Eq. (9). In this regard, the Red Pitaya-related energy consumption is assumed to be uniform during the signal collection phase, whose duration is equal to $\Delta t_{\text {COLLECTION }}$. 


$$
e=\operatorname{Power}(\text { RedPitaya }) \cdot \Delta t_{\text {COLLECTION }}+e_{\text {TRAIN }}
$$

To compare the PZT with the EMAT configuration, the corresponding energy consumption needs to be evaluated. Once we derived that, the corresponding maximum transmission frequency compatible with the battery back availability criterion $\left(f^{\max }\right)$ needs to be derived, i.e. the power consumption needs to be such that the system conditions can be continuously monitored for 72 hours. At this point, if $f^{\max }$ is lower than $f_{\text {safety }}$, the corresponding technology cannot be adopted, Eq. (10).

$$
f^{\max } \geq f_{\text {safety }}
$$

In particular, if the considered technology-related energy consumption $\left(e_{E M A T}\right.$ and $\left.e_{P Z T}\right)$ is such that the maximum frequency which allows monitoring the system for 72 hours is lower than $f_{\text {safety }}$, it means that the energy consumption is too high and it does not allow to get system updated every minute, and then the technology is not suitable for this purpose.

\subsection{Communication channel losses}

Estimates of energy required to operate the communication system consist, in part, of estimates of expected losses incurred by the acoustic signal during transmission along the pipe. Considering the case of transmission along a straight section of a pipe, losses in the channel include absorption and diffraction of acoustic beam. Diffraction causes acoustic beam spot to diverge relative to initial value at the transducer. Because the receiver samples acoustic waves only through a limited area, a significant fraction of the energy is lost. Active element of paintbrush PZT has a rectangular shape. Therefore, we use the model of diffraction of a beam through a rectangular aperture.

\subsubsection{Diffraction losses}

The propagation problem in free space can be formulated using the coordinate system where $\mathrm{z}$ is the direction of propagation, and $\rho$ is the transverse plane radius. The acoustic field at the output of the transmitting PZT is $U_{i}\left(\rho_{1}\right)$ in the $\mathrm{z}=0$ plane, and the acoustic field at the receiving PZT is $U_{o}\left(\rho_{2}\right)$ in the $\mathrm{z}=L$ plane. Fraunhofer diffraction formula under the paraxial approximation in the far field gives

$$
U_{o}\left(\rho_{2}\right)=\frac{e^{i k\left(L+\left|\rho_{2}\right|^{2} / 2 L\right)}}{i \lambda L} \widetilde{U}_{i}\left(\rho_{2} / \lambda L\right)
$$

Where $\widetilde{U}_{i}\left(\rho_{2} / \lambda L\right)$ is the Fourier transform of the input field $U_{i}\left(\rho_{1}\right)$ evaluated at the spatial frequency $\vec{f}=\bar{\rho}_{2} / \lambda L$. The spatial Fourier transform is defined as

$$
\widetilde{U}(f)=\int_{-\infty}^{\infty} d \rho U(\rho) e^{-i 2 \pi f \cdot \rho}
$$




$$
U(p)=\int_{-\infty}^{\infty} d f \tilde{U}(f) e^{i 2 \pi f \cdot p}
$$

The acoustic field of the transmitting PZT can be written as

$$
U_{i}\left(\rho_{1}\right)=\operatorname{Urect}\left(x_{1} / d_{1}\right) \operatorname{rect}\left(y_{1} / d_{2}\right)
$$

Where

$$
\operatorname{rect}(x)= \begin{cases}1 & |x| \leq 1 / 2 \\ 0 & |x|>1 / 2\end{cases}
$$

With the Fourier transform given as

$$
F . T .\{\operatorname{rect}(x)\}=\sin c\left(f_{x}\right)=\frac{\sin \left(\pi f_{x}\right)}{\pi f_{x}}
$$

The transmitted power can be calculated as

$$
P_{t}=\int d x_{1} \int d y_{1}\left|U_{i}\left(\rho_{1}\right)\right|^{2}=|U|^{2} d_{1} d_{2}
$$

The acoustic field at the receiving PZT at $\mathrm{z}=L$ plane can be expressed as

$$
U_{o}\left(\rho_{2}\right)=\frac{U e^{i k\left(L+\left|\rho_{2}\right|^{2} / 2 L\right)}}{i \lambda L} d_{1} d_{2} \sin c\left(\frac{d_{1} x_{2}}{\lambda L}\right) \sin c\left(\frac{d_{2} y_{2}}{\lambda L}\right)
$$

The intensity at the receiving PZT can be given as

$$
I_{0}\left(\rho_{2}\right)=\left|U_{o}\left(\rho_{2}\right)\right|^{2}=\frac{|U|^{2} d_{1}^{2} d_{2}^{2}}{\lambda^{2} L^{2}} \sin c^{2}\left(\frac{d_{1} x_{2}}{\lambda L}\right) \sin c^{2}\left(\frac{d_{2} y_{2}}{\lambda L}\right)
$$

The power crossing the aperture of the receiving PZT at with the same dimensions $d_{1}$ and $d_{2}$ is given as

$$
\begin{aligned}
P_{r} & =\int_{-d_{1} / 2}^{d_{1} / 2} d x_{2} \int_{-d_{2} / 2}^{d_{2} / 2} d y_{2} I_{0}\left(\rho_{2}\right)= \\
& =\frac{|U|^{2} d_{1}^{2} d_{2}^{2}}{\lambda^{2} L^{2}} \int_{-d_{1} / 2}^{d_{1} / 2} d x_{2} \int_{-d_{2} / 2}^{d_{2} / 2} d y_{2} \sin c^{2}\left(\frac{d_{1} x_{2}}{\lambda L}\right) \sin c^{2}\left(\frac{d_{2} y_{2}}{\lambda L}\right)
\end{aligned}
$$


The integral can be calculated approximately by noting that $d_{1}^{2} / 2 \lambda L \ll 1$ and $d_{2}^{2} / 2 \lambda L \ll 1$, so that the value of each integral is approximately $d_{1}$ and $d_{2}$, respectively. Thus, the received power is

$$
P_{r} \approx \frac{|U|^{2} d_{1}^{2} d_{2}^{2}}{\lambda^{2} L^{2}} d_{1} d_{2}=P_{t} \frac{d_{1}^{2} d_{2}^{2}}{\lambda^{2} L^{2}}=\frac{P_{t}}{\left(\lambda L / d_{1}^{2}\right)\left(\lambda L / d_{2}^{2}\right)}
$$

The power transmission coefficient is

$$
T=\frac{P_{r}}{P_{t}}=\frac{1}{\left(\lambda L / d_{1}^{2}\right)\left(\lambda L / d_{2}^{2}\right)}
$$

The transmission coefficient in $\mathrm{dB}$ units cab be written is

$$
S_{21}=20 \log _{10}\left(\frac{d_{1} d_{2}}{\lambda L}\right)
$$

This equation is valid for acoustic wave transmission a through medium at normal incidence. Next, we consider diffraction loss for a wave propagating at oblique angle. A schematic diagram of the setup is shown in Figure 4. Longitudinal acoustic wave is launched from a PZT, propagates through the acrylic contoured surface wedge. Because the wedge angle is larger than the first critical angle, the refracted wave is shear-only. The shear wave is supported inside metal if the acoustic wavelength is smaller than the metal pipe wall thickness.

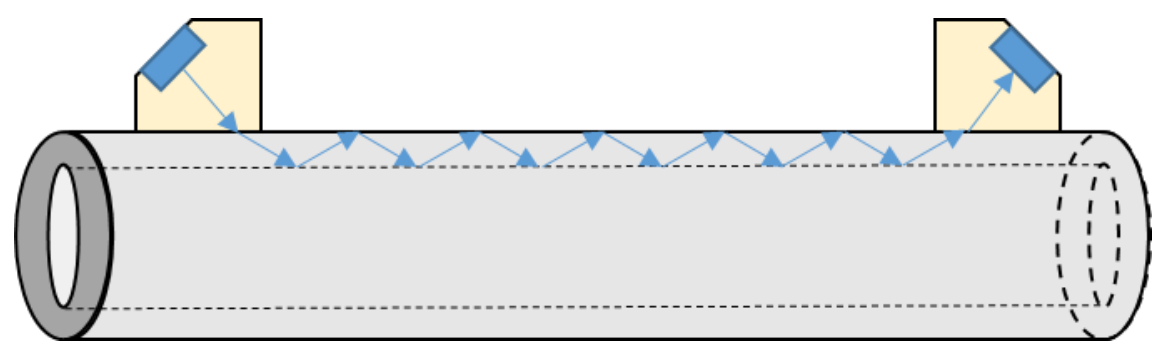

Figure 4. Schematic diagram of acoustic signal transmission.

Expanded diagram of the acoustic wave refraction is shown in Figure 5. Angles of the incident longitudinal and refracted shear waves are related according to acoustic Snell's Law

$$
\frac{\sin \theta_{1}}{V_{L_{1}}}=\frac{\sin \theta_{2}}{V_{S_{2}}}
$$

Where $\mathrm{V}_{\mathrm{L} 1}$ is the speed of the longitudinal wave in the acrylic wedge, $\mathrm{V}_{\mathrm{S} 2}$ is the speed of shear wave in the metal, $\theta_{1}$ and $\theta_{2}$ are the incident and refracted wave angles. Inside the metal, 
reflection angle of the acoustic shear wave is $\theta_{2}$. The description assumes that wavelength is smaller than the thickness of metal, so that geometric optics approximation is valid

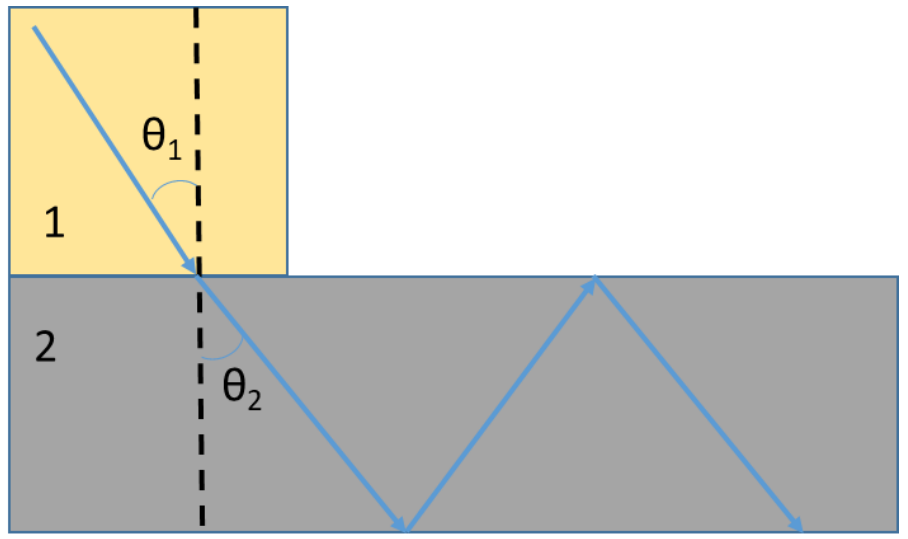

Figure 5. Schematic diagram of acoustic wave refraction and shear wave propagation in metallic plate.

Note that the distance along the pipe and the actual distance travelled by the acoustic shear wave differ by a factor of $1 / \sin \theta_{2}$. That is, if the distance along the pipe is $\mathrm{L}$, the distance travelled by the shear wave is $\mathrm{L} / \sin \theta_{2}$. Combining (13) and (14), and using $\lambda=\mathrm{V}_{\mathrm{S} 2} / \mathrm{f}$ where $\mathrm{f}$ is the acoustic frequency, we obtain

$$
S_{21}=20 \log _{10}\left(\frac{d_{1} d_{2} f \sin \theta_{1}}{V_{L_{1}} L}\right)
$$

Note that (25) does not include any pipe parameters.

\subsubsection{Comparison with Experiment}

Based on parameter values of CVCS nuclear-grade pipe, a stainless steel schedule-160 pipe with parameter values listed in Table 3 was used for proof-of-principle studies of acoustic signal transmission.

Table 3. Geometrical parameters of stainless steel pipe.

\begin{tabular}{cc}
\hline $\begin{array}{c}\text { Pipe } \\
\text { Parameters }\end{array}$ & Values \\
\hline Length & 6 feet \\
\hline OD & 2.375 in \\
\hline Wall & 0.344 in \\
Thickness & $(8.7 \mathrm{~mm})$ \\
\hline Type & Schedule-160 \\
\hline
\end{tabular}

Two 500kHz PZT's were mounted on $30^{\circ}$ acrylic wedges countered for the pipe shape. The first critical angle is $27.6^{\circ}$, so that the refracted wave for $30^{\circ}$ incidence is shear wave-only A relatively 
low acoustic frequency of $500 \mathrm{kHz}$ was chosen to minimize coupling losses. Values of longitudinal and shear wave velocities, as well as the values of incident and calculated value of refracted shear wave angle are listed in Table 4.

Table 4. Acoustic wave parameter values

\begin{tabular}{cc}
\hline Parameter & Value \\
\hline $\mathrm{V}_{\mathrm{L} 1}$ & $\begin{array}{c}2680 \\
(\mathrm{~m} / \mathrm{s})\end{array}$ \\
\hline $\mathrm{V}_{\mathrm{S} 2}$ & $\begin{array}{c}3100 \\
(\mathrm{~m} / \mathrm{s})\end{array}$ \\
\hline$\theta_{1}$ & $30^{\circ}$ \\
\hline$\theta_{2}$ & $35^{\circ}$ \\
\hline $\mathrm{f}$ & $480 \mathrm{KHz}$ \\
\hline$\lambda$ & $6.5 \mathrm{~mm}$ \\
\hline $\mathrm{d}_{1}$ & $1.1875 \mathrm{in}$ \\
\hline $\mathrm{d}_{2}$ & $0.625 \mathrm{in}$ \\
\hline
\end{tabular}

Although the nominal PZT frequency is $500 \mathrm{kHz}$, experiment showed that resonant frequency for signal transmission in the assembled system was $480 \mathrm{kHz}$. Corresponding shear wave wavelength is $\lambda=6.5 \mathrm{~mm}$, which is smaller than the wall thickness of $8.7 \mathrm{~mm}$. Therefore, criteria for geometric-optic model validity discussed above are satisfied. Values of $\mathrm{d}_{1}$ and $\mathrm{d}_{2}$ of the paintbrush PZT parameters listed in Table 3 are those of the wedge aperture in the plane of contact with the pipe. The numbers are slightly larger than the nominal PZT aperture of 1 in $x$ $0.5 \mathrm{in}$. Note that for the values in Table 3 , the criteria of the far field $d^{2} / \lambda \ll L$ is satisfied for L>1foot.

In the validation experiment, PZT was chirped with $10 \mathrm{kHz}$ bandwidth around $480 \mathrm{kHz}$ center frequency, and the received signal was integrated over the bandwidth. One PZT was coupled to pipe with gel, while the other one was dry-coupled. The dry-coupled PZT was moved along the pipe to measure the signal at various distances. Experimental values were obtained for $\mathrm{L}=3,4$, and 5 feet PZT to PZT distance. Comparison of calculated and measured signal values is shown in Figure 4. The calculated signal is obtained by simulating Eq. (25) with the set of parameter values in Table 4. 


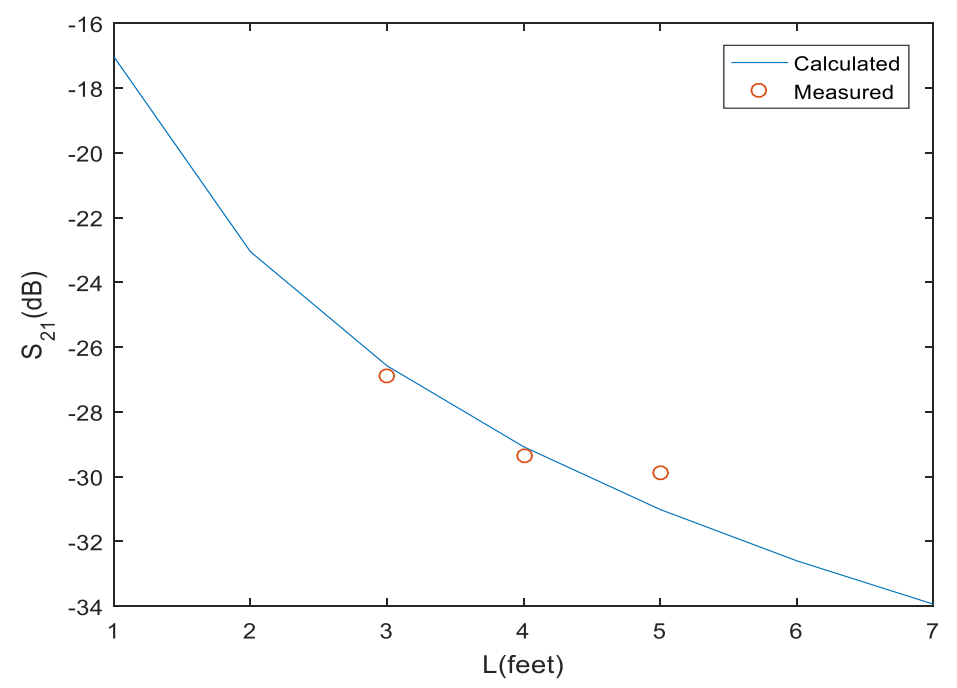

Figure 6. Comparison of calculated and measured acoustic shear wave transmitted signals.

Because diffraction was assumed to be the only source of signal loss in the model, good agreement between model and observations indicates that, aside from transducer coupling, acoustic diffraction is the main source of signal loss.

\section{SOFTWARE DEFINED ULTRASONIC COMMUNICATION SYSTEM}

The objective of this section is to assess the capability of using ultrasonic waves as a carrier waveform for communications. In this study, a ZYNQ System-on- Chip (SoC) platform is used to conduct ultrasonic communication experiments. This system consists of a transmitting transducer and a receiving transducer operating at $\mathrm{MHz}$ range frequencies. The transceiver unit operates using In-phase and Quadrature (IQ) modulation. With IQ modulation, different modulation schemes such as quadrature amplitude modulation (QAM), phase shift keying (PSK) can be readily implemented on the platform without modifying the hardware setup. Experiments are conducted in solid channels with various shape, material and size to explore the ultrasonic communication feasibility in diverse environments. Performance of different modulation methods are discussed and compared in this paper.

\subsection{Introduction}

In this study, piezoelectric transducers are used to generate and receive ultrasonic signals. A platform based on the Field Programmable Gate Array (FPGA), high speed data and signal converters, amplifiers, and transducers are assembled to conduct ultrasonic communication experiments. Experiments are conducted in solid materials with different channel type, shape, and sizes. The objective is to design and examine a reliable system for the ultrasonic communication in solids having diverse physical and geometrical configurations [9].

A critical processing component of SDR is In-phase and Quadrature (IQ) modulation. IQ modulation is an efficient way to transfer information and is easier to be implemented on the digital platform such as FPGA. Also, with IQ modulation, one can implement a variety of 
Analog and Digital modulation methods. Figure 7 shows the block diagram of the IQ modulation and de-modulation.

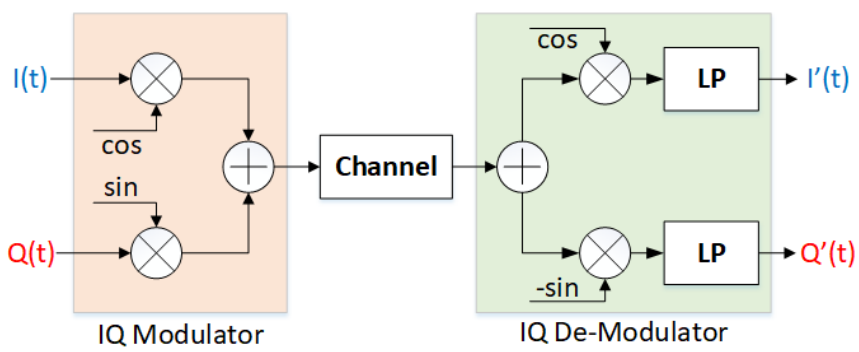

Figure 7. IQ Modulator and De-Modulator block diagrams

In IQ modulation, in-phase component is multiplied by cosine carrier and quadrature component is multiplied by sinusoid carrier. The modulated signal is the summation of these two products. The de-modulation procedure is found by multiplying the received signal with both sinusoid and cosine waves generated by a local oscillator at the same frequency. This will shift the baseband signal from carrier frequency back to low frequency. It will then be filtered by a low-pass filter to recover the information.

\subsection{Hardware Platform}

To conduct ultrasonic communication experiments, we considered a ZYNQ based Red-Pitaya system which is capable of sampling and reconstructing signals at 125 MSPS. This system consists of two ADCs and two DACs which makes it ideal for SDR based ultrasonic communication in $\mathrm{MHz}$ frequency range. Both high speed $\mathrm{ADC}$ and DAC can operate at 125 MSPS, and have a $3 \mathrm{~dB}$ bandwidth of $50 \mathrm{MHz}$ and resolution of 14 bits. ZYNQ SoC manufactured by Xilinx consists of dual core ARM A9 processors and a FPGA fabric. The FPGA serving as the peripheral of the ARM processor can expand the system IO and accelerate the algorithm realization. The high bandwidth between the FPGA and ARM processor allows the user to design high performance reconfigurable systems. 


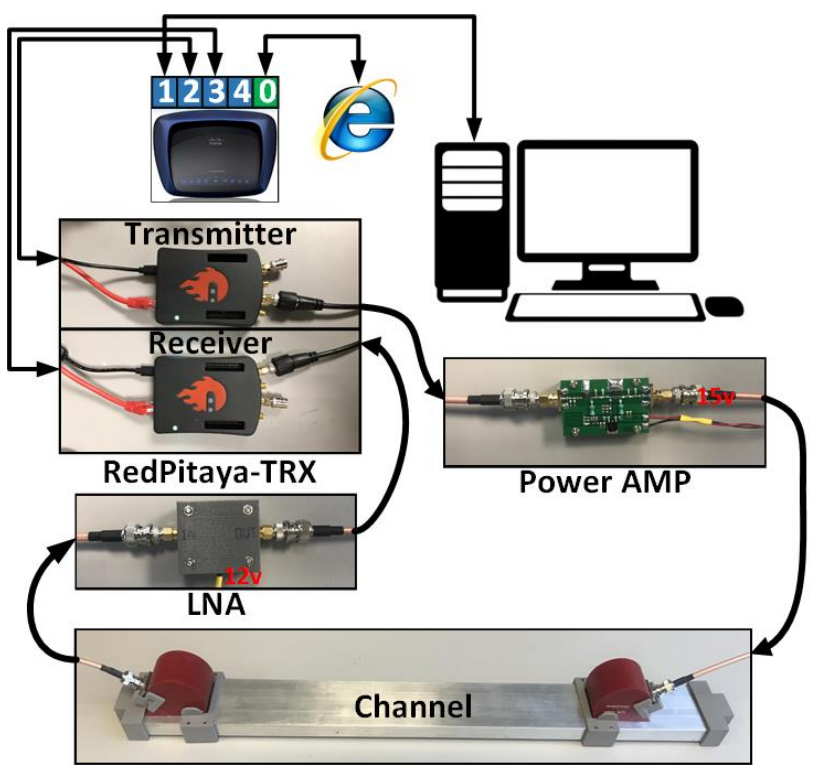

Figure 8. Ultrasonic communication system diagram.

Figure 8 displays the system diagram of the ultrasonic communication experimental platform. The system contains two Red-Pitaya boards, both are configured as transceivers which have full capability of transmitting and receiving RF signals up to $60 \mathrm{MHz}$. In this test setup, the upper device is used as the transmitter. The baseband signal generated by the host computer will be sent to a transmitter through the ethernet cable. On the FPGA, this baseband signal is processed by a digital up-converter (DUC) and sent to the on-board 125 MSPS DAC. The output is connected to a power amplifier to increase the driving capability. The amplified signal will be converted to an ultrasonic wave by a piezoelectric transducer and travel through the channel. The signal will be picked up by the receiving transducer. Due to attenuation, reflection and refraction in the channel, the received signal is weak and must be amplified. Therefore, the received signal is amplified by a $20 \mathrm{~dB}$ Low Noise Amplifier (LNA) and sampled at $125 \mathrm{MSPS}$ ADC. The sampled signal is processed using a digital down-converter on the FPGA to recover the baseband signal.

Figure 9 shows the block diagram of the Digital Up Converter (DUC) and the Digital Down Converter (DDC) on the FPGA. The red arrows in the figure represents high speed AXI4-Stream bus interface. Green arrows represent AXI4-Lite bus interface, it is a general purpose read and write bus interface which allows for low speed data transmission between peripheral and processor. The cascaded integrator-comb (CIC) compiler is a Xilinx LogiCORE IP core which provides the ability to design and implement AXI4-Stream-compliant CIC filters [10]. CIC multi-rate filters are commonly used for implementing large sample rate changes in digital systems. In SDR systems, the carrier frequency is much higher than the baseband frequency. A CIC filter is necessary for both DUC and DDC subsystems to match the sample frequency of the carrier signal and baseband signal. The Direct Digital Synthesizer (DDS) compiler is a Xilinx LogiCORE IP core which is used to generate precise Sinusoid and Cosine waveforms for DUC and DDC [11]. The DUC implements I/Q modulator and the DDC implements the I/Q demodulator according to the diagram shown in Figure 1. 


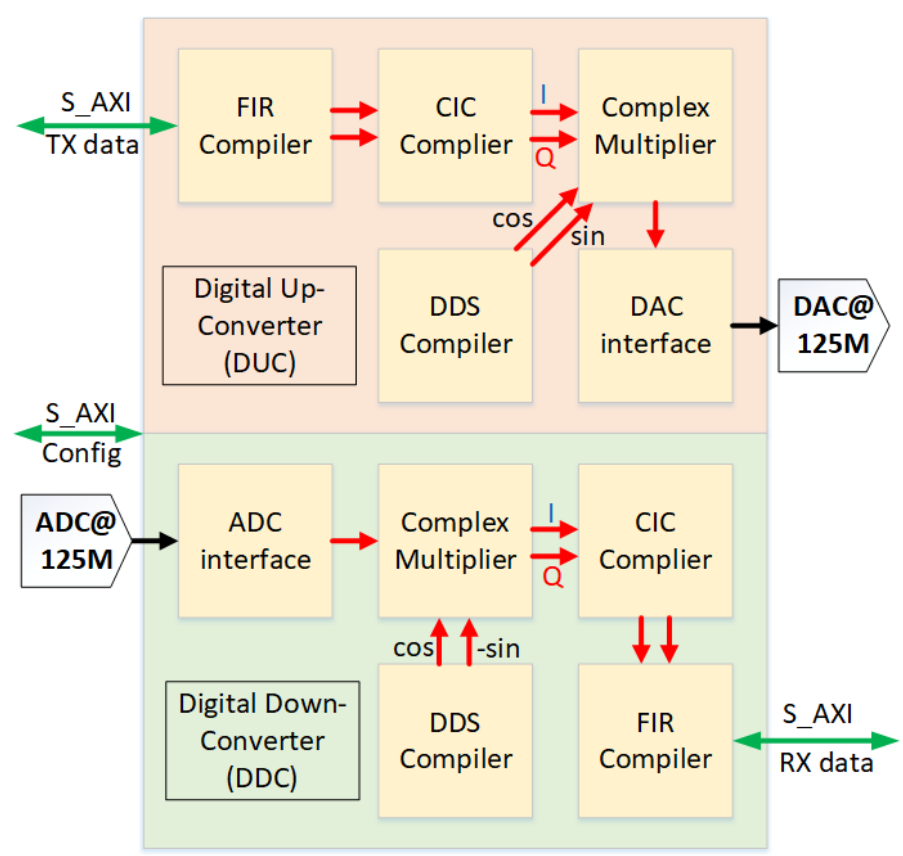

Figure 9. Block diagram of FPGA

Figure 9 also shows the benefit of using a ZYNQ SoC for the SDR platform. The ARM processor is used to generate and receive the low frequency baseband signals through the AXILite interface, and the FPGA fabric on-chip will handle most of the heavy computations including DUC and DDC. The FPGA also makes it possible to connect multiple high-speed ADCs and DACs without adding additional peripheral and circuits. The high bandwidth between the ARM processor and FGPA allows for real-time communication and add expandability to the system. In previously published papers [12], [13], the efficiency and reconfigurability of using ZYNQ SoC for high speed signal processing system design is presented.

\subsection{Experiments and Results}

PZT transducer is used as the energy transformer to convert the electric energy to ultrasonic wave. The PZT transducer can generate and receive ultrasonic waves at its resonant frequency. Figure 10 shows a practical test setup for conducting ultrasonic communication. With the support of a 3D printed holder, PZT transducers are mounted on the two sides of the acrylic column. Ultrasonic coupling gel with medium viscosity is used on the contact surface of the acrylic column and on the PZT transducers to ensure a better transmission and reception of the ultrasonic signals. With this test setup bulk waves are generated for ultrasonic communications under ideal conditions. 


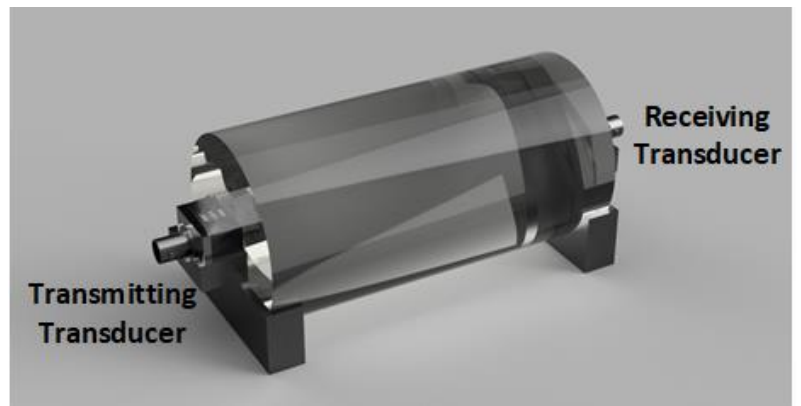

Figure 10. Test Setup A - Transmitting and receiving with transducers at the ends of a column

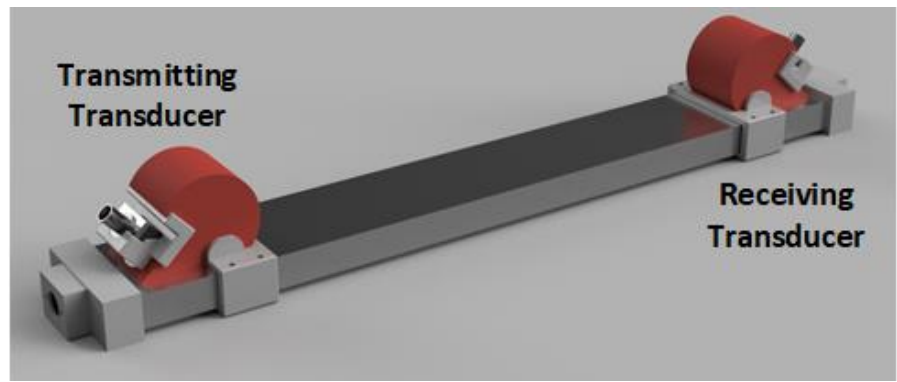

Figure 11. Test Setup B - Transmitting and Receiving with transducers at oblique angles

In Figure 11, the ultrasonic signal is transmitted through a solid bar with oblique angle wedges. 3D printed sliding fixtures are used to move the transducers along the solid bar. Two $2.5 \mathrm{MHz}$ piezoelectric transducers are used for transmitting and receiving ultrasonic signals. Both wedges are 60-degree. The distance between the two transducers is defined by the point where the main beam of ultrasonic signal is supposed to enter the metal bar to the point where the receiver transducer should pick it up.

Figure 12 shows the modulation and de-modulation of a $312 \mathrm{kbps}$ baseband signal modulated using on-off keying (OOK) method. In this scenario, the quadrature component is set to 0 and in-phase component is either 0 or 1 . Figure $12 \mathrm{~b}$ shows the received signal. Figure $12 \mathrm{c}$ is the demodulated IQ data obtained by using the DDC on the FPGA. The Test Setup A (see Figure 4) is utilized, and the channel is acrylic column (3-inch diameter and 3-inch length). Two $2.5 \mathrm{MHz}$ PZT transducers are used as the transmitter and the receiver.

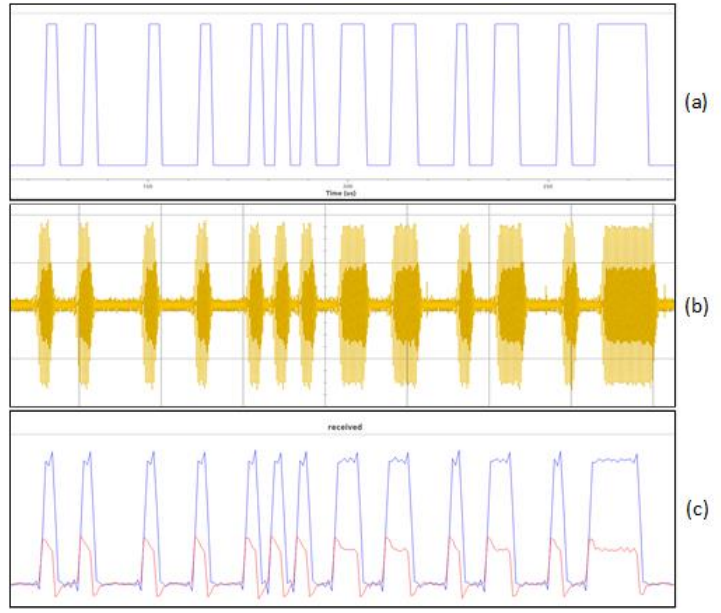

Figure 12. Simple OOK transmitting and receiving. 


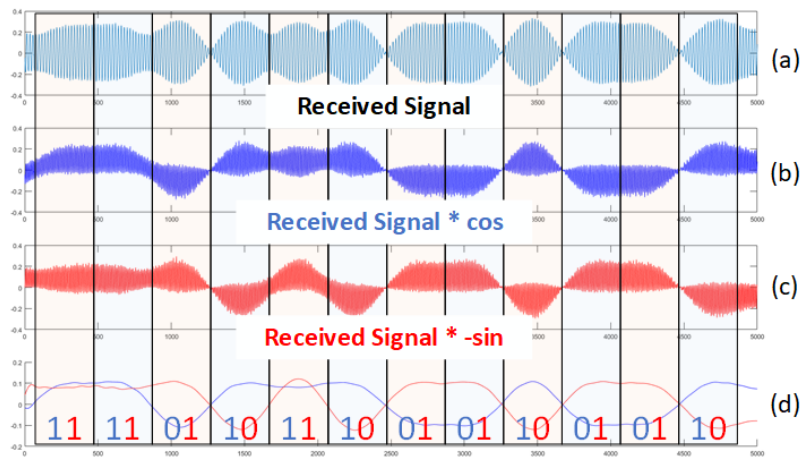

Figure 13. QPSK de-modulation procedures.

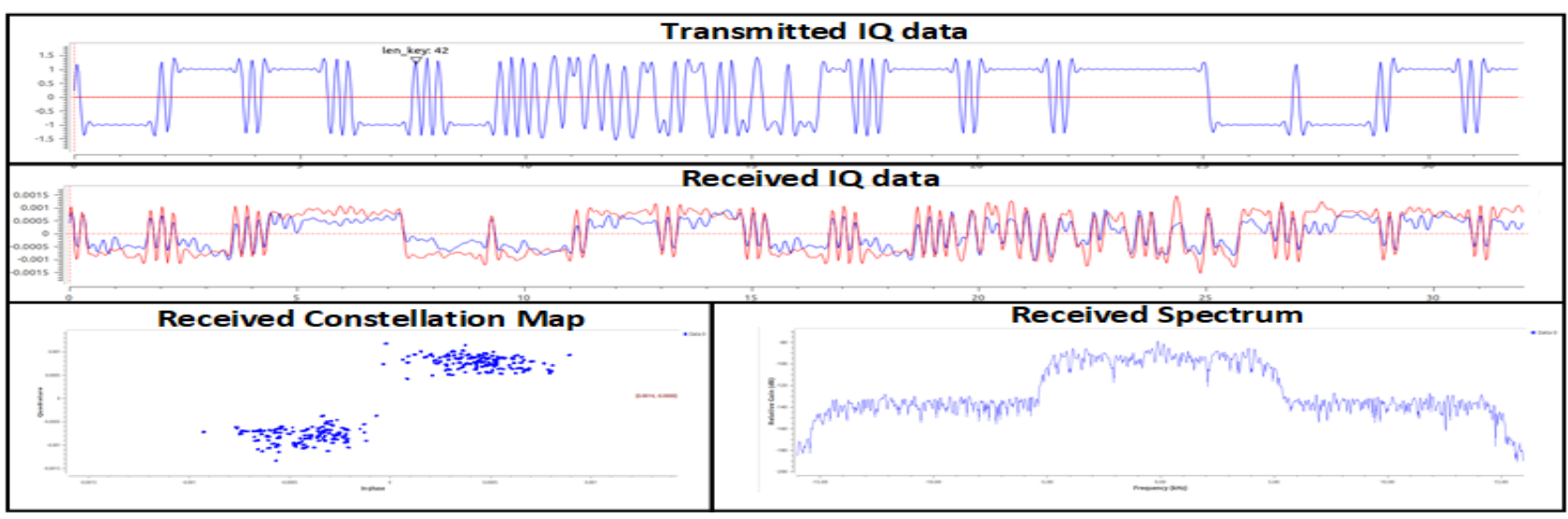

Figure 14. BPSK transmitting and receiving.

Figure 13 shows the transmitting and receiving signal using the binary phase shift keying (BPSK) when the Test Setup B (see Figure 11) is used. In this setup, the quadrature component is set to 0 and in-phase component is either -1 or 1 . The distance between the two transducers is set to 0.5 meter. As expected, the BPSK modulation scheme offered the most robust performance for conducting communications using ultrasonic signals.

\subsection{Conclusions and Future Work}

This section discussed the feasibility and advantages of using ultrasonic signal for communication in a diverse environment when other options for information transmission are not feasible. A Software Defined Radio (SDR) system that can be used for ultrasonic communications is introduced. This system is based on ZYNQ SoC (includes FPGA Fabric and ARM Processors) for information processing. Digital up converter and down converters on FPGA are used to process the high frequency signal in real-time. On-chip ARM processor will generate and receive the baseband signal. High speed ADC and DAC are used to transmit and receive RF ultrasonic signals. With this SDR platform, we can conduct a series of experiments with different modulation techniques, and various type of transducers and channel setups. 


\section{EMAT-BASED ULTRASONIC COMMUNICATION SYSTEM}

In this study, EMAT is used as the transmitter for non-contact ultrasonic signal generation and PZT is used as the receiver for the communication system. This system is designed to assess the efficiency of Lamb waves and to examine Bit Error Rate (BER) of digital communication. The communication system was configured using National Instrument arbitrary waveform generator (AWG) and PXI digital oscilloscope. The accuracy of transmitted information is tested on a 5foot long stainless-steel plate and pipe. The system operates with the frequency of $414 \mathrm{kHz}$ signal and uses Amplitude-Shift Keying (ASK) modulation for transmission. This method can transfer digital data in presence of undesirable multipath effect with bit rates of $20 \mathrm{k}$ bps and 40k bps without any error.

\subsection{Introduction}

In conventional ultrasonic communication systems using solid materials as the communication channel, a piezoelectric (PZT) transducer is used as the transmitter of information and the trustworthiness of the received information is highly dependent on the coupling condition between the PZT transducer and the communication channel [9], [14]. EMAT (Electro Magnetic Acoustic Transducer) as an alternative transducer to PZT, which has not been utilized for communications because of higher power excitation and offers low transduction efficiency [15]. In addition, with high power excitation, EMAT to EMAT communication may induce large RF coupling interference, which adds noise to acoustic communication [13], [14]. However, in harsh environments (hot, cold, sealed or contaminated), the noncontact EMAT holds promise for the transmission of information by acoustic means in solids. In practice, the conventional ultrasonic communications techniques which employ contact PZT transducers [15] may suffer from the ambiguity in signal quality due to uncertainty in a coupling condition. In contrast, EMAT is non-contact transduction [16] and becomes a promising choice for transmitting information in solids such as pipes and plates. In this paper, we present a practical solution using EMAT as a noncontact transmitter and PZT as a receiver. This system configuration can compensate the disadvantage of EMAT to EMAT RF coupling interference. To keep the quality of the signal that can propagate through a 5-foot stainless steel plate or pipe with less attenuation [17], we select the central frequency of the signal to be $500 \mathrm{kHz}$. For the study an experimental platform with programmable arbitrary waveform generator and digital data acquisition system is designed to test and optimize the performance of the communication system. The results are compared and analyzed.

\subsection{EMAT Theory}

EMAT can transform the electromagnetic energy into acoustic energy. It consists of a magnet providing the static magnetic field, a metallic sample providing the medium of theultrasonic signal, and a coil inducing alternating currents. In metallic materials, the ultrasonic signal is generated by Lorentz forces. While in ferromagnetic material, the ultrasonic signal is generated by the Lorentz force, the magnetization force and the magnetostrictive force [18], [19]. In this paper, the communication channels are metallics and made of stainless-steel plate and pipe where the Lamb waves are generated by Lorentz force. 
The transduction of EMAT is based on Lorenz force $F_{l}$, which is the cross product of the Eddy current $J$ induced by the electric coil and static magnetic field $B_{0}$ near the surface of a metallic material. This process is usually described as

$$
F_{l}=J \times B_{0}
$$

Depending on the geometry of electric coils and the permanent magnet, different modes, such as SH (Shear Horizontal) wave, SV (Shear Vertical) wave, and Lamb waves can be generated. Figure 15. shows the structure and geometry of EMAT Lamb wave generator.

Lamb wave is similar to surface wave except that they can only be generated in thin materials only a few wavelengths thick. So, the incident angle of the lamb wave generated by EMAT is $90^{\circ}$ and it propagates along a plate or pipe surface. In this paper, the thickness of the stainlesssteel pipe and plate is $9 \mathrm{~mm}$ and $9.5 \mathrm{~mm}$ respectively, which is around 1.5 times of wavelength. Lamb waves are complex vibrational waves that propagate parallel to the material surface throughout the thickness of the material. The size of the meander coils determines the working frequency range and wave frequency and thickness determines the modes [20] generated by EMAT. After implementing the frequency sweep program, which is introduced in next section, the carrier frequency is selected as $414 \mathrm{kHz}$.

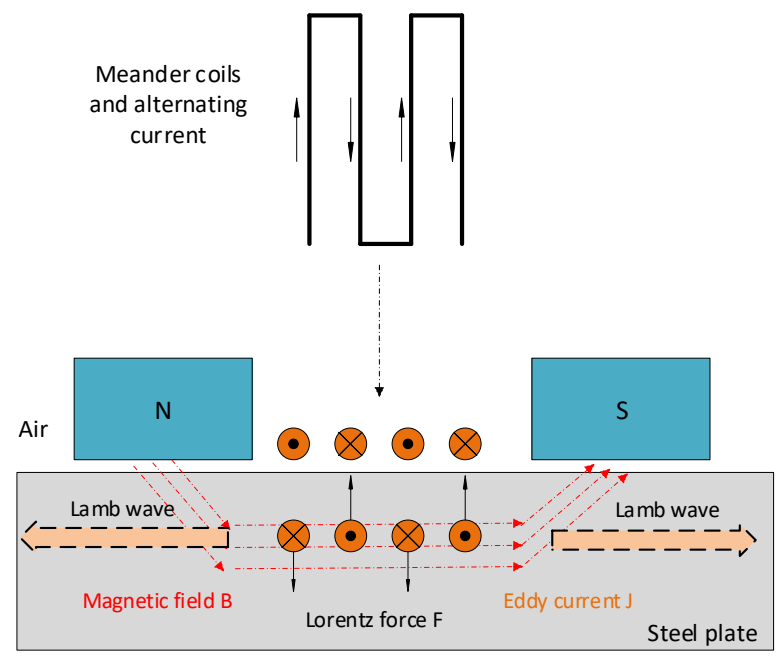

Figure 15. EMAT geometry for Lamb wave generation.

\subsection{System Description and Experimental Setup}

The communication platform developed for this study consists of EMAT and PZT Transducers, a binary message generator, a power amplifier for EMAT excitation, a low noise pre-amplifier attached to the PZT receiving transducer, a data acquisition unit and two application programs: Frequency Sweep Program and Communication Test Program.

\subsubsection{System Configuration}

The EMAT used in the experiment is composed of an XXL size magnet (274A0093-M00) and RF meander coils from INNERSPEC. The recommended frequency range for this EMAT is $200 \mathrm{kHz}$ to $500 \mathrm{kHz}$. A $500 \mathrm{kHz}$ PZT transducer with angle wedge is utilized as the receiver. 
Figure 16 shows the schematic of the laboratory communication system. The system is assembled using an arbitrary waveform generator (AWG), PXI oscilloscope, a power amplifier with 50dB fixed gain and a low-noise pre-amplifier. The low noise pre-amplifier has a build in band-pass filter that can improve the SNR of the received signal. The digital information (binary code) is modulated using the amplitude shift keying (ASK) for transmission through a solid channel. The PXI oscilloscope will record the modulated signal and received signal detected by the PZT receiver. The PC will store the data and make it available for signal processing and analysis.

In this study, two channels are used to propagate the ultrasonic signal for performance evaluation. Figure 17 (a) shows a $169 \mathrm{~cm}(5.4 \mathrm{feet})$ long, $10 \mathrm{~cm}$ wide and $0.95 \mathrm{~cm}$ thick stainlesssteel plate that is used as a communication channel. Figure 17(b) shows a $194 \mathrm{~cm}$ (6.04 feet) long, $6.1 \mathrm{~cm}$ outer radius and $4.1 \mathrm{~cm}$ inner radius steel pipe is used as a communication channel. The distance between EMAT transmitter and PZT ultrasonic receiver is set to 1.54 meters ( 5 feet).

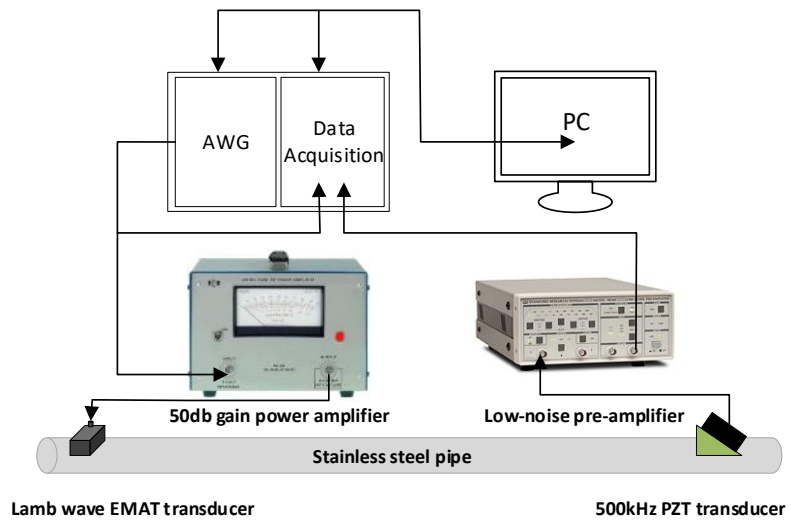

Figure 16. Schematics of laboratory electronics setup.

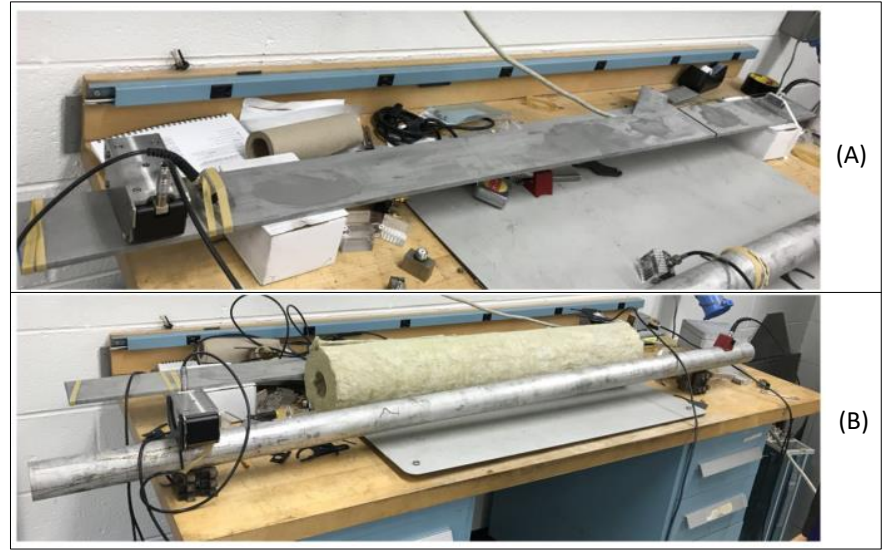

Figure 17. Communication over stainless steel channels (a) Plate. (b) Pipe.

\subsubsection{Program Description}

The Frequency Sweep Program can help to determine the best frequency response of the EMAT transmitter and the PZT receiver. This program sweeps the frequency and then display the 
frequency response of the signals. We can set the transmitted and received signal acquisition time, frequency sweep steps, acquisition delay and sampling rate, which are shown in the control panel of Figure 18. However, the frequency response fluctuates significantly due to the complex modes of the Lamb waves. The aim is to determine a desirable carrier frequency with power concentrated in one mode. The signals displayed in Figure 18 are the transmitted signal and transmitted spectrum, received signal and received spectrum, and the received signal for optimized frequency detected in $200 \mathrm{kHz}$ to $600 \mathrm{kHz}$ frequency range. For the current pair of transducers, the optimized frequency is within the $405 \mathrm{kHz}$ to $420 \mathrm{kHz}$ frequency range. Based on the experimental results, $414 \mathrm{kHz}$ is selected as the carrier frequency for ASK communications.

ASK shown in Equation (17) is a modulation process, which imparts a sinusoid wave with certain frequency into two discrete amplitude levels. These are related to the value of the binary code representing the digital message.

$$
s(t)=\left\{\begin{array}{rr}
A \cos \left(2 \pi f_{c} t\right), & \text { binary } 1 \\
0, & \text { binary } 0
\end{array}\right.
$$

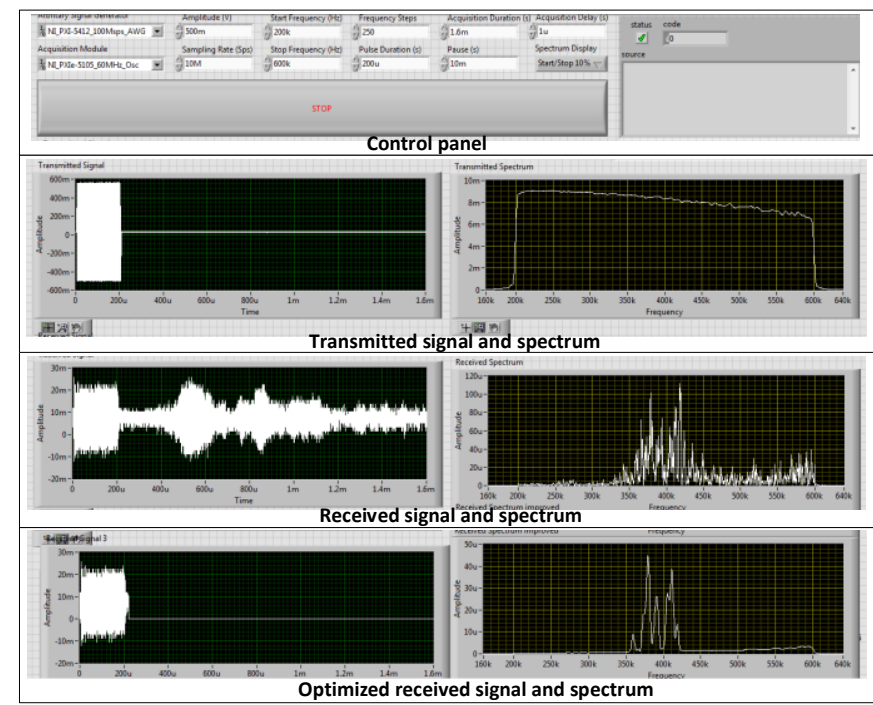

Figure 18. Frequency sweep program interface.

A coherent demodulation method is implemented to recover the transmitted and received binary waveforms for comparison. This comparison is for bit error detection and estimation of bit error rate (BER).

Figure 19 shows the front panel of the Communication Test Program. The program can send 1-1000 bits of binary code that can be random or fixed. With the setting of the pulse duration and number of bits, different bit durations can be selected. For example, if 10 bits are transmitted and the pulse duration is $1 \mathrm{~ms}$, bit duration is $100 \mathrm{us}$ which implies the system can communicate with a data rate of $10 \mathrm{kbps}$.

The Communication Test Program can show the power spectrum in linear or dB scale. The acquisition delay setting is to synchronize the transmitted and received signals. As mentioned in the previous section, the distance between two transducers is 1.54 meters, the delay depends on 
the Lamb wave velocity. Lamb wave velocity is determined by the thickness of the channel and carrier frequency. Different modes of Lamb wave velocity can be found in the Lamb wave phase dispersion curve [20], [21]. As a result, the setting of delay varied with different channels and carrier frequency. After demodulating the raw data captured by the receiver, the received binary waveform can be converted into binary code. The received binary code is compared with the transmitted binary code to detect and display errors in the test program front panel. For example, Figure 19 shows the input binary code (0010101010 and the bit duration (100 microseconds), the system fully recovers the transmitted binary code and the bit error rate is 0 .

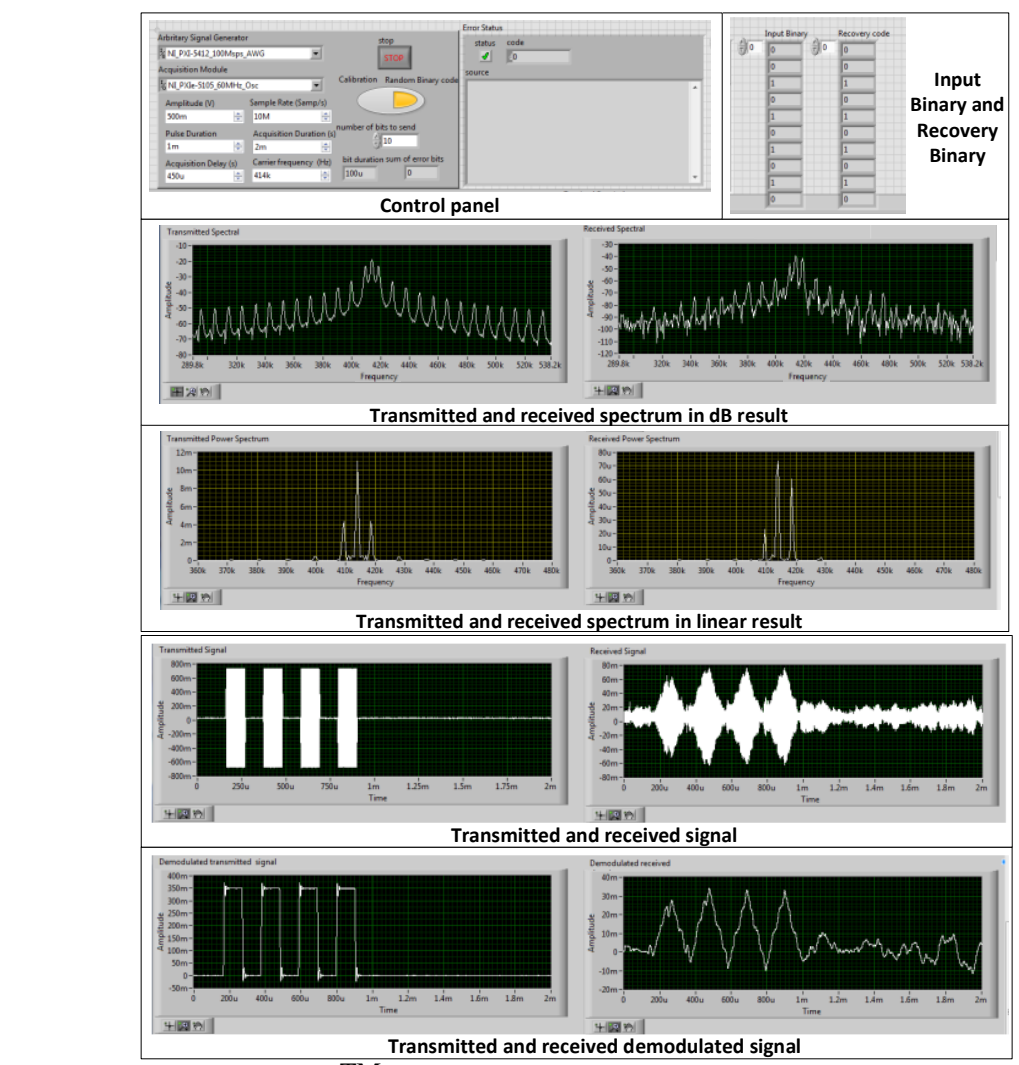

Figure 19. Labview ${ }^{\mathrm{TM}}$ interface of communication system program.

\subsection{Data Analysis}

To test the EMAT signal, and to estimate the velocity of the Lamb wave, experiments are performed using the plate and pipe channels with different transducer-to-transducer distances. In the experiment (see Figure 20), the position of receiver is fixed, and distance between the receiver and the transmitter is varied: $145 \mathrm{~cm}, 120 \mathrm{~cm}, 95 \mathrm{~cm}, 70 \mathrm{~cm}$ and $35 \mathrm{~cm}$. The received signals using the plate and pipe channels are shown in Figure 21. and Figure 22. 


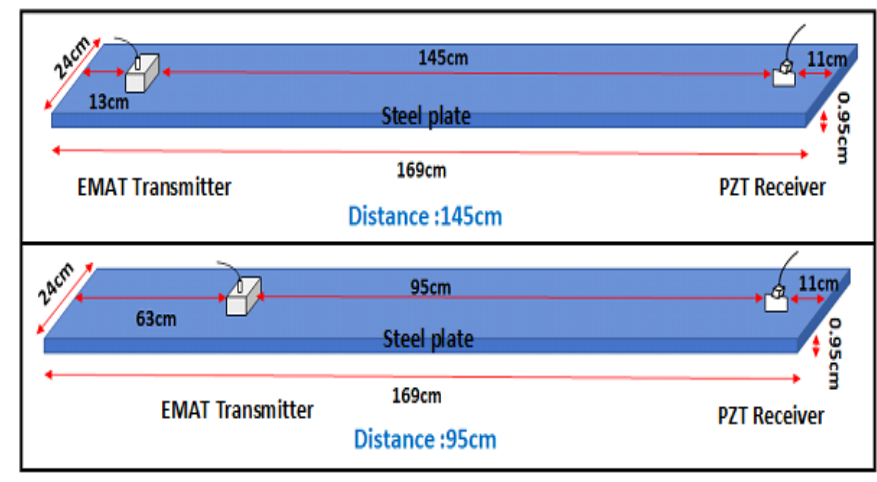

Figure 20. Schematics of two test cases on a plate.

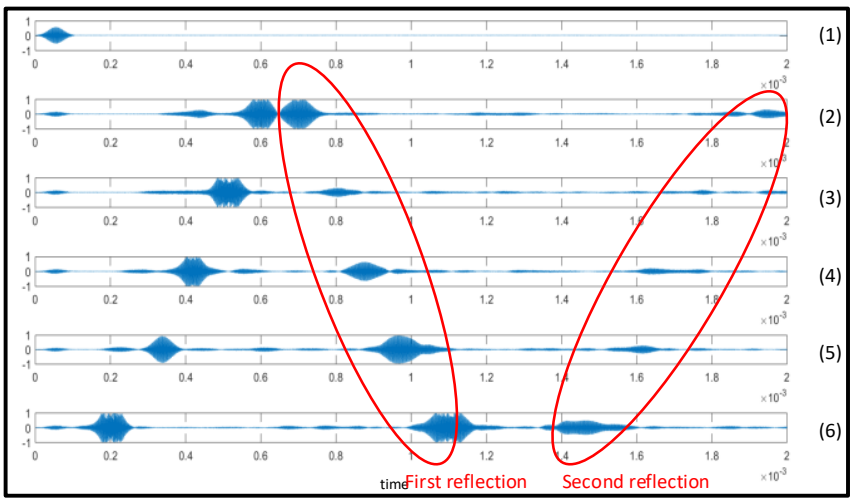

Figure 21. Test results for the plate channel (1) transmitted signal (2) received signal when receiver is $145 \mathrm{~cm}$ away from transmitter (3) received signal when receiver is $120 \mathrm{~cm}$ away from transmitter (4) received signal when receiver is $95 \mathrm{~cm}$ away from transmitter (5) received signal when receiver is $70 \mathrm{~cm}$ away from transmitter (6) received signal when receiver is $35 \mathrm{~cm}$ away from transmitter

Figure 21 shows the results of an experiment implemented on the steel plate, with ultrasonic carrier frequency of 414k. The transmitted signal is a100us bit duration Gaussian signal. The RF coupling interference between the transmitter and receiver is insignificant compared with the actual received ultrasonic signal. Several modes exist in the received signal and most of the energy stays on one mode which shows up around 600us (see Figure 21). The first plate-edge reflection of the received signal shows up late since the transmitter is far from the edge. Since the Lamb wave propagates along the plate, the velocity of each signal can be estimated. Velocity estimations are shown in Table 5. There are about $10 \%$ variation in calculating the velocity for distance $70 \mathrm{~cm}$ and $35 \mathrm{~cm}$ because the actual distance does not consider the signal travelling length within the receiving wedge. The average velocity is $2594 \mathrm{~m} / \mathrm{s}$, which matches the Lamb wave phase velocity dispersion. 
Table 5. Lamb wave velocity estimates

\begin{tabular}{|c|c|c|c|c|c|}
\hline $\begin{array}{l}\text { Distance } \\
\text { Transmitter } \\
\text { to Receiver }\end{array}$ & $145 \mathrm{~cm}$ & $120 \mathrm{~cm}$ & $95 \mathrm{~cm}$ & $70 \mathrm{~cm}$ & $35 \mathrm{~cm}$ \\
\hline $\begin{array}{l}\text { Distance } \\
\text { Transmitter } \\
\text { to edge }\end{array}$ & $13 \mathrm{~cm}$ & $38 \mathrm{~cm}$ & $63 \mathrm{~cm}$ & $88 \mathrm{~cm}$ & $123 \mathrm{~cm}$ \\
\hline $\begin{array}{l}\text { First peak } \\
\text { signal arrival } \\
\text { time }\end{array}$ & 600 us & 500 us & 410us & 340us & 210us \\
\hline $\begin{array}{l}\text { Second peak } \\
\text { signal arrival } \\
\text { time }\end{array}$ & 720us & 800 us & 880 us & 960us & 1100 us \\
\hline $\begin{array}{l}\text { Third peak } \\
\text { signal arrival } \\
\text { time }\end{array}$ & 1900us & 1820us & 1710us & 1620us & 1500us \\
\hline $\begin{array}{l}\text { Speed of first } \\
\text { peak signal }\end{array}$ & $2636 \mathrm{~m} / \mathrm{s}$ & $2667 \mathrm{~m} / \mathrm{s}$ & $2639 \mathrm{~m} / \mathrm{s}$ & $2413 \mathrm{~m} / \mathrm{s}$ & $2188 \mathrm{~m} / \mathrm{s}$ \\
\hline $\begin{array}{l}\text { Speed of } \\
\text { second peak } \\
\text { signal }\end{array}$ & $2552 \mathrm{~m} / \mathrm{s}$ & $2614 \mathrm{~m} / \mathrm{s}$ & $2663 \mathrm{~m} / \mathrm{s}$ & $2703 \mathrm{~m} / \mathrm{s}$ & $2676 \mathrm{~m} / \mathrm{s}$ \\
\hline $\begin{array}{l}\text { Speed of } \\
\text { third peak } \\
\text { signal }\end{array}$ & $2515 \mathrm{~m} / \mathrm{s}$ & $2478 \mathrm{~m} / \mathrm{s}$ & $2515 \mathrm{~m} / \mathrm{s}$ & $2554 \mathrm{~m} / \mathrm{s}$ & $2515 \mathrm{~m} / \mathrm{s}$ \\
\hline
\end{tabular}

The test results, which are shown in Figure 22 for the pipe channel, are similar to those of the plate channel. In the received signal, the first reflection and second reflection are still visible. However, more modes show up due to the special geometry of the pipe. Received signals with different bit duration have different patterns compared with the test results using the plate channel. Since the thickness of plate and pipe are different, their velocity is different too. The average estimated velocity is $2859 \mathrm{~m} / \mathrm{s}$.

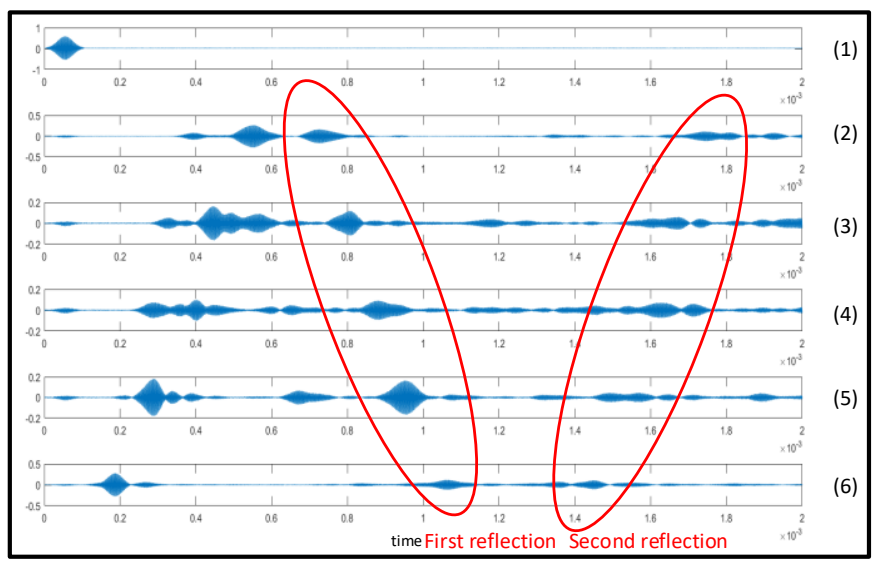

Figure 22. Test results for the pipe channel (1) transmitted signal (2) received signal when receiver is $145 \mathrm{~cm}$ away from transmitter (3) received signal when receiver is $120 \mathrm{~cm}$ away from transmitter (4) received signal when receiver is $95 \mathrm{~cm}$ away from transmitter $\mathrm{m}(5)$ received signal when receiver is $70 \mathrm{~cm}$ away from transmitter (6) received signal when receiver is $35 \mathrm{~cm}$ away from transmitter.

After obtaining the Lamb wave velocity with a $414 \mathrm{kHz}$ carrier frequency through the plate and pipe channels, the delay setting can be determined (593 microseconds for plate channel and 539 microseconds for pipe channel). In the experiment the distance between the transducers is $154 \mathrm{~cm}$ and the transmitted signal is set to 001010101010 bits binary code. The experimental 
results are shown in Figures 23 and 24. These results indicate that the delay setting is correct and can keep the transmitter and receiver synchronized. For the plate channel, the received signal can recover the binary code with bit duration from 100 microseconds to 25 microseconds which corresponds to $10 \mathrm{kps}$ to $40 \mathrm{kbps}$. For pipe channel, the system can recover the bit duration from 100 microseconds to 50 microseconds and there will be error bit with 33 microseconds bit duration. The bit duration 25 microseconds cannot be recovered in the communication system. The results can prove that using EMAT as a transmitter for the communication system is feasible and efficient.

\subsection{Conclusion}

We have conducted preliminary studies of the feasibility of using EMAT for ultrasonic communications. Benchtop electronic system based on AWG and oscilloscope was assembled. With this test system, proof-of-principle ultrasonic communication studies were conducted with EMAT as a transmitter and PZT as receiver for data transmission using 5-foot steel plate and pipe channels. The usage of these two types of transducers guarantees that EMAT can be excited with less power and low RF coupling interference. ASK modulation method is adapted to modulate the baseband signal at the rate of $40 \mathrm{kbps}$ across the plate channel and $20 \mathrm{kbps}$ across the pipe channel. The Lamb wave mode selection is done by the frequency sweep program. The signal of $414 \mathrm{kHz}$ carrier frequency shows the best frequency response in the spectrum. The velocity of the Lamb wave mode is calculated. The system is carefully designed to minimize bit error rate to ensure robust communication.

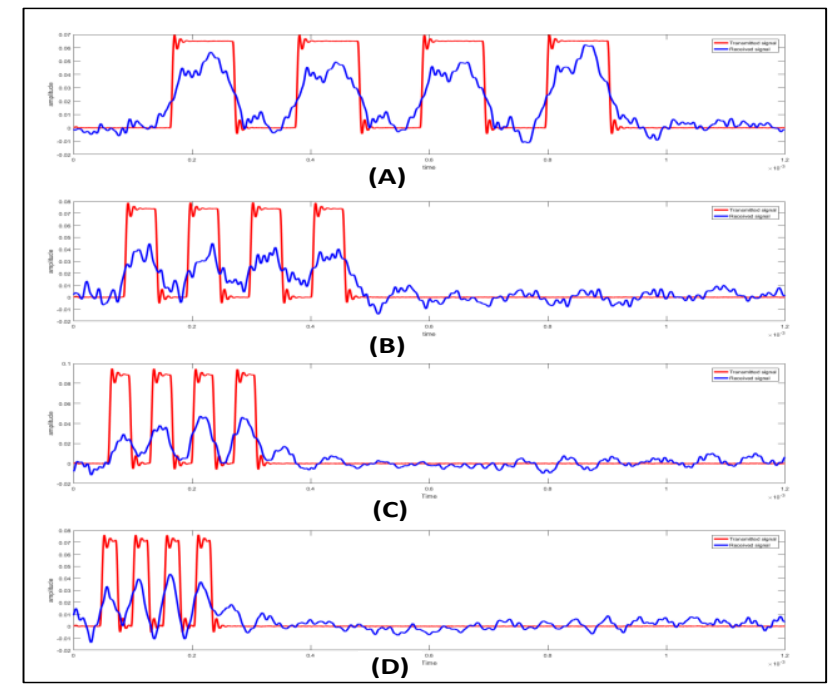

Figure 23. Transmitted and received signals with the plate channel (a) Bit duration of 100 microseconds (b) Bit duration of 50 microseconds (c) Bit duration of 33 microseconds (d) Bit duration of 25 microseconds 


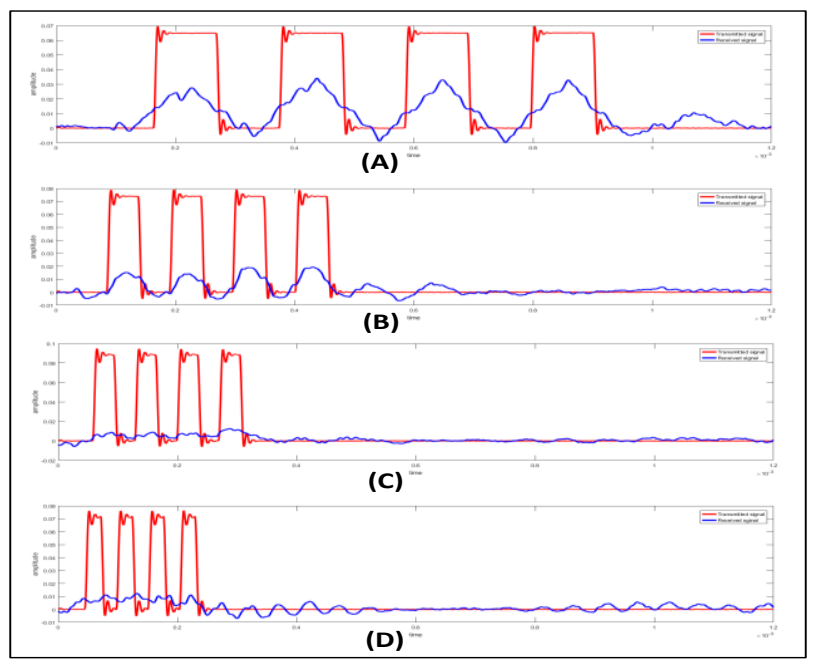

Figure 24. Transmitted and received signals with the pipe channel (a) Bit duration of 100 microseconds (b) Bit duration of 50 microseconds (c) Bit duration 33 microseconds (d) Bit duration of 25 microseconds.

\section{CONCLUSION}

The completed tasks reported here lay the ground work for next phase of ultrasonic EMAT-based communication system development. The analysis performed in Section 2 indicates that despite higher power consumption, EMAT would be preferable transducer to PZT for nuclear facility information transmission applications. The study described in Section 3 establishes the results for ultrasonic communication system in software-defined radio environment. The results reported in Section 4, indicate feasibility of communication using EMAT. Future efforts on the project will consider frequency modulation (FM) information encoding, in addition to amplitude and phase shift keying schemes. 


\section{REFERENCES}

[1] A. Heifetz, S. Bakhtiari, X. Huang, R. Ponciroli and R.B. Vilim, "First Annual Progress Report on Transmission of Information by Acoustic Communication along Metal Pathways in Nuclear Facilities," ANL/NE-17/30, September 30, 2017.

[2] R. Kolbe and E. Gahan, "Survey of Insulation Used in Nuclear Power Plants and the Potential for Debris Generation," NUREG/CR-2403, SAND82-0927 Supplement No.1 (1982).

[3] B.S. Lee, R. Travis, E. Grove, and A. Di Biasio, "Aging Assessment of Westinghouse PWR and General Electric BWR Containment Isolation Functions," NUREG/CR-6339, BNLNUREG-52462, Brookhaven National Laboratory, 1996.

[4] K. Korsah, P. Ramuhalli, R. Vlim, R. A. Kisner, C.L. Britton Jr., D.W. Wootan, N.C. Anheier Jr., A.A. Diaz, E.H. Hirt, H.T. Chien, S. Sheen, S. Bakhtiari, S. Gopalsami, A. Heifetz, S.W. Tam, Y. Park, B.R. Upadhyaya, A. Stanford, "Assessent of sensor technologies for advanced reactors," ORNL/TM-2016/337, October 2016.

[5] R. Vilim, A. Heifetz, "Dynamic stability of the S-CO2 energy conversion cycle as affected by sensor response time," Proceedings of ICAPP (2016).

[6] A. Heifetz and R. Vilim, "Eigendecomposition model of resistance temperature detector with applications to S-CO 2 cycle sensing," Nuclear Engineering and Design 311, 60-68 (2017).

[7] B.S. Hoffheins, T.E. McKnight, R.J. Lauf, R.E. James, and R.R. Smith, "Evaluation of hydrogen sensor for nuclear reactor containment monitoring," American Nuclear Society 1997 Advanced Reactor Safety Meeting, Orlando (Florida), June 1-4, 1997.

[8] T.M. Cover and J.A. Thomas, Elements of Information Theory, $2^{\text {nd }}$ Ed., Wiley 2006.

[9] B. Wang, J. Saniie, S. Bakhtiari and A. Heifetz, "Architecture of an ultrasonic experimental platform for information transmission through solids," 2017 IEEE International Ultrasonics Symposium (IUS), Washington, DC, pp. 1-4, 2017.

[10] Xilinx, "CIC Compiler v4.0 LogiCORE IP Product Guide," 5 OCT 2016. [Online].Available:https://www.xilinx.com/support/documentation/ip_documentation/cic_ compiler/v4_0/pg140-cic-compiler.pdf. [Accessed 26 Feb 2018].

[11] Xilinx, "DDS Compiler v6.0 LogiCORE IP Product Guide," 20 Dec 2017. [Online].Available:https://www.xilinx.com/support/documentation/ip_documentation/dds _compiler/v6_0/pg141-dds-compiler.pdf. [Accessed 26 Feb 2018].

[12] B. Wang and J. Saniie, "Ultrasonic Signal Acquisition and Processing platform based on Zynq SoC,” 2016 IEEE International Conference on Electro Information Technology (EIT), pp. 448-451, 2016.

[13] B. Wang, P. Govindan and J. Saniie, "Performance Analysis of System-on-Chip Architectures for Ultrasonic Data Compression," 2016 IEEE International Ultrasonics Symposium (IUS), pp. 1-4, 2016.

[14] S. Chakraborty, G.J. Saulnier, K.W. Wilt, E. Curt, H. A. Scarton, R.B. Litman, "Low-power, low-rate ultrasonic communications system transmitting axially along a cylindrical pipe using transverse waves," IEEE Transactions on Ultrasonics, vol. 62, no. 10, pp. 1788-1796, 2015. 
[15] N. K. Mutlib, S. B. Baharom, A. EI-Shafie and M. Z. Nuawi, "Ultrasonic health monitoring in structural engineering: buildings and bridges," Strucural Control Health Monitoring, vol. 23, no. TOC, pp. 409-422, 2015.

[16] E. Dobbs and J. Llewellyn, "Generation of ultrasonic waves without using a transducer," Non-Destructive Testing, vol. 4, no. 1, pp. 49-56, 1971.

[17] F. Schubert, "Numberical time-domain modeling of linear and nonlinear ultrasonic wave propagation using finite integration techniques-theory and applications," Ultrasonics, vol. 42, no. 1-9, pp. 221-229, 2004.

[18] H. Ogi, "Field dependence of coupling efficiency between electromagnetic field and ultrasonic bulk waves," Journal of Applied Physics, vol. 82, no. 8, pp. 3940-3949, 1997.

[19] R. Thompson, "A model for the electromagnetic generation of ultrasonic guided waves in ferromagnetic metal polycrystals," IEEE Transactions on Sonics and Ultrasonics, vol. 25, no. 1, pp. 7-15, 1978.

[20] K. M. Riichi Murayama, "Conventional electromagnetic acoustic transducer development for optimum Lamb wave modes," Ultrasonics, vol. 40, no. 1-8, pp. 491-495, 2002.

[21] B. C. G. T. Borja Sorazu, "Obtaining complementary Lamb wave dispersion information by two signal processing methods on an all-optical non-contact configuration," SENSORS ACTUATORS, vol. 217, pp. 95-104, 2014. 
Argonne

Nuclear Engineering Division

Argonne National Laboratory

9700 South Cass Avenue, Bldg. 208

Argonne, IL 60439

www.anl.gov 